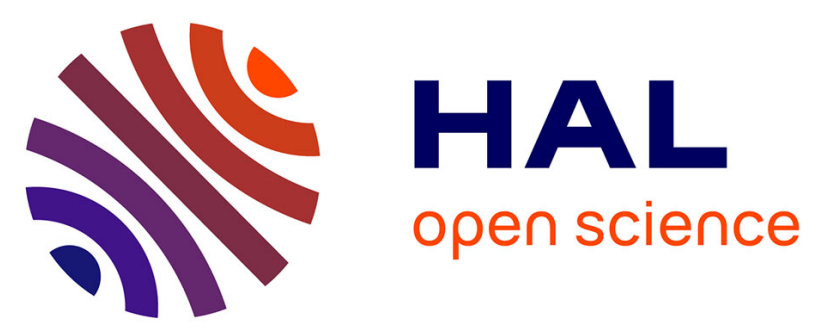

\title{
How to: Measuring blood cytokines in biological psychiatry using commercially available multiplex immunoassays
}

\author{
Raoul Belzeaux, Marie-Noëlle Lefebvre, Anne Lazzari, Tifenn Le Charpentier, \\ Julia-Lou Consoloni, Xavier Zendjidjian, Mocrane Abbar, Philippe Courtet, \\ Jean Naudin, José Boucraut, et al.
}

\section{To cite this version:}

Raoul Belzeaux, Marie-Noëlle Lefebvre, Anne Lazzari, Tifenn Le Charpentier, Julia-Lou Consoloni, et al. How to: Measuring blood cytokines in biological psychiatry using commercially available multiplex immunoassays. Psychoneuroendocrinology, 2017, 75, pp.72-82. 10.1016/j.psyneuen.2016.10.010 . hal-01575039

\section{HAL Id: hal-01575039 \\ https://hal-amu.archives-ouvertes.fr/hal-01575039}

Submitted on 17 Aug 2017

HAL is a multi-disciplinary open access archive for the deposit and dissemination of scientific research documents, whether they are published or not. The documents may come from teaching and research institutions in France or abroad, or from public or private research centers.
L'archive ouverte pluridisciplinaire HAL, est destinée au dépôt et à la diffusion de documents scientifiques de niveau recherche, publiés ou non, émanant des établissements d'enseignement et de recherche français ou étrangers, des laboratoires publics ou privés. 
Psychoneuroendocrinology

Elsevier Editorial System(tm) for

Manuscript Draft

Manuscript Number: PNEC-D-15-00657R3

Title: How to: Measuring blood cytokines in biological psychiatry using commercially available multiplex immunoassays

Article Type: Research paper

Section/Category: Clinical science

Keywords: Cytokine, Psychiatry, Inflammation, Multiplex immunoassay

Corresponding Author: Dr. El Chérif Ibrahim, Ph.D.

Corresponding Author's Institution: Aix Marseille Université-CNRS

First Author: Raoul Belzeaux

Order of Authors: Raoul Belzeaux; Marie-Noëlle Lefebvre; Anne Lazzari; Tifenn Le Carpentier; Julia-Lou Consoloni; Xavier Zendjidjian; Mocrane Abbar; Philippe Courtet; Jean Naudin; José Boucraut; Pierre Gressens; Nicolas Glaichenhaus; El Chérif Ibrahim, Ph.D.

Abstract: Cytokines produced by both immune and non-immune cells are likely to play roles in the development and/or progression of psychiatric disorders. Indeed, many investigators have compared the blood cytokine levels in psychiatric patients with those of healthy controls or monitored their levels in patients during disease progression to identify biomarkers. Nevertheless, very few studies have confirmed that such cytokines remain stable in healthy individuals through periods of weeks and months. This is an important issue to consider before using blood cytokine levels as biomarkers of disease traits, disease state, or treatment response. Although multiplex assay technology represents an advance in identifying biomarkers because it allows simultaneous examination of large panels of analytes from a small volume of sample, it is necessary to verify whether these assays yield enough sensitivity and reproducibility when applied to the blood from neuropsychiatric patients. Therefore, we compared two multiplex immunoassays, the bead-based Luminex® (Bio-Rad) and the electro-chemiluminescence-based V-plex® (MesoscaleDiscovery), for the detection and quantification of 31 cytokines, chemokines and growth factors in both the sera and plasma of patients with major depressive episodes (MDE) and age- and sex-matched healthy control subjects during a 30-week period. Although both platforms exhibited low coefficients of variability (CV) between the duplicates in the calibration curves, the linearity was better in general for the VPLEX® platform. However, neither platform was able to detect the absolute values for all of the tested analytes. Among the 16 analytes that were detected by both assays, the intra-assay reproducibility was in general better with the V-PLEX® platform. Although it is not a general rule that the results from sera and plasma will be correlated, consistent results were more frequent with the V-PLEX® platform. Furthermore, the V-PLEX® results were more consistent with the gold standard ELISA simplex assay for IL-6 in both sera and plasma. The intra-individual variability of the measurements, among the sera and plasma for the 4 samples harvested from 
each healthy individual, was low for Eotaxin, G-CSF, IL-4, IL-7, IL-9, IL-12p40, IL-12p70, IL-15, MIP-1 $\beta, \mathrm{PDGF}-\mathrm{BB}, \mathrm{TNF}, \mathrm{TNF}-\beta$ and VEGF, but intermediate or high for IFN-Y, IL-6, IL-8, IL-10, and IP10. Together, these data suggest that extreme caution is needed in translating the results of multiplex cytokine profiling into biomarker discovery in psychiatry. 


\title{
El Chérif Ibrahim, PhD
}

Aix-Marseille Université, CNRS, CRN2M-UMR 7286

Faculté de Médecine Nord de Marseille

13344 Marseille Cedex 15, FRANCE

Tél : +33(0)491698956

el-cherif.ibrahim@univ-amu.fr

\author{
Beate Ditzen \\ Guest Editor of \\ Psychoneuroendocrinology
}

Dear Editor,

We thank you and the Editor in chief, Robert Dantzer, for reaching the conclusion to ultimately publish our manuscript. Following your suggestion, we changed the tiltle to: "How to: Measuring blood cytokines in biological psychiatry using commercially available multiplex immunoassays".

We were concerned by the persistent negative comments of Reviewer\#3, stressing out that our comparison between the two platforms we have worked with suffers a number of theoretical and technical limitations because of the difference in the number of analytes that are measured by each platform and the lack of assessment of the between assay variability. Therefore, and following your recommandations, we made as clear as possible in the discussion section the limits of our study, and we also introduced some new conclusions supported by new results we just obtained this month:

"Our findings have several important technical and theoretical limitations. First, we could not compare exactly the same sets of cytokines on both platforms on the basis of the commercial availability, and it has previously been observed that assay performance decreases with increasing numbers of markers on a panel (Chaturvedi et al., 2011). It will be important in the future to follow up with studies to compare larger sets of cytokines as similar to each other as possible, because recent investigations in the field of biomarker discovery have revealed new candidates for promising proteins. Secondly, we did not address the inter-assay variability on each platform, which is an important factor to consider especially when investigating large samples that cannot be processed with a single batch of reagents or for longitudinal studies when assaying 
the samples at different days over long periods of time. Third, our results should be considered to be preliminary on the basis of the relatively small sample size that makes any group comparison between healthy control and patients inappropriate. Of note, for the purpose of another study, all serum samples assayed in the present study, and obtained from healthy control and MDE patients at their first visit, have been tested again on the MSD platform with a different batch of reagents and a two-year interval (data not shown). The same panels of cytokines were assayed as well as an additional chemokine panel. A comparative analysis of old and new measurements of cytokine concentrations revealed robust correlations for IFN- $\gamma, I L-6, I L-8, I L-10, I L-$ 12p40, IL-15, IL-16, TNF, TNF- $\beta$, and VEGF (data not shown), suggesting a strong inter-assay stability for these analytes on the MSD platform. Moreover, the new measurements for IP-10 and MIP-1 13 obtained on the MSD platform strongly correlated with previous measurements obtained with the Luminex platform (data not shown), suggesting that these assays exhibit low intra- and inter-assay variability on both platforms.

With the hope of a final approval of our manuscript in Psychoneuroendocrinology.

Sincerely,

\section{El Chérif IBRAHIM}


How to: Measuring blood cytokines in biological psychiatry using commercially available multiplex immunoassays

Raoul Belzeaux ${ }^{1,2,3}$, Marie-Noëlle Lefebvre ${ }^{4}$, Anne Lazzari ${ }^{5}$, Tifenn Le Carpentier ${ }^{6,7}$, Julia-Lou Consoloni ${ }^{1,2,3}$, , Xavier Zendjidjian², Mocrane Abbar ${ }^{8}$, Philippe Courtet ${ }^{3,9}$, Jean Naudin², José Boucraut $^{1,3,10}$, Pierre Gressens ${ }^{6,7}$, Nicolas Glaichenhaus ${ }^{5}$, El Chérif Ibrahim ${ }^{1,3}$

${ }^{1}$ Aix-Marseille Univ, CNRS, CRN2M-UMR7286, Marseille, France

${ }^{2}$ Pôle de Psychiatrie, Assistance Publique-Hôpitaux de Marseille, Marseille, France

${ }^{3}$ Fondation FondaMental, Fondation de Recherche et de Soins en Santé Mentale, Créteil, France

${ }^{4}$ CIC-CPCET, AP-HM, Hôpital La Timone, Marseille, France

${ }^{5}$ Institut de Pharmacologie Moléculaire et Cellulaire CNRS, INSERM, Université de NiceSophia Antipolis Valbonne, France

${ }^{6}$ Inserm, U1141, Paris, France

${ }^{7}$ Univ Paris Diderot, Sorbonne Paris Cité, UMRS 1141, Paris, France

${ }^{8}$ Pôle de Psychiatrie, Centre Hospitalier Régional Universitaire Carémeau, Nîmes, France

${ }^{9}$ Département des Urgences et Post-Urgences Psychiatriques, Centre Hospitalier Régional Universitaire, Hôpital Lapeyronie, Montpellier, France

${ }^{10}$ Laboratoire d'Immunologie, AP-HM, Hôpital de la Conception, Marseille, France

\section{Corresponding author:}

El Chérif Ibrahim, 51 Bd Pierre Dramard, 13344 Marseille Cedex 15 France

Tel: +33 (0)4 916989 56; Fax: +33 (0)4 91698920

Email: el-cherif.ibrahim@univ-amu.fr 


\section{Abstract}

Cytokines produced by both immune and non-immune cells are likely to play roles in the development and/or progression of psychiatric disorders. Indeed, many investigators have compared the blood cytokine levels in psychiatric patients with those of healthy controls or monitored their levels in patients during disease progression to identify biomarkers. Nevertheless, very few studies have confirmed that such cytokines remain stable in healthy individuals through periods of weeks and months. This is an important issue to consider before using blood cytokine levels as biomarkers of disease traits, disease state, or treatment response. Although multiplex assay technology represents an advance in identifying biomarkers because it allows simultaneous examination of large panels of analytes from a small volume of sample, it is necessary to verify whether these assays yield enough sensitivity and reproducibility when applied to the blood from neuropsychiatric patients. Therefore, we compared two multiplex immunoassays, the bead-based Luminex® (Bio-Rad) and the electro-chemiluminescence-based V-plex® (MesoScaleDiscovery), for the detection and quantification of 31 cytokines, chemokines and growth factors in both the sera and plasma of patients with major depressive episodes (MDE) and age- and sex-matched healthy control subjects during a 30-week period. Although both platforms exhibited low coefficients of variability $(\mathrm{CV})$ between the duplicates in the calibration curves, the linearity was better in general for the V-PLEX® platform. However, neither platform was able to detect the absolute values for all of the tested analytes. Among the 16 analytes that were detected by both assays, the intra-assay reproducibility was in general better with the V-PLEX® platform. Although it is not a general rule that the results from sera and plasma will be correlated, consistent results were more frequent with the V-PLEX® platform. Furthermore, the V-PLEX® results were more consistent with the gold standard ELISA simplex assay for IL-6 in both sera and plasma. The intra-individual variability of the measurements, among the sera and plasma for the 4 samples harvested from each healthy individual, was low for Eotaxin, G-CSF, IL-4, IL-7, IL-9, IL-12p40, IL-12p70, IL-15, MIP-1 $\beta$, PDGF-BB, TNF, TNF- $\beta$ 
R. Belzeaux et al.

and VEGF, but intermediate or high for IFN- $\gamma$, IL-6, IL-8, IL-10, and IP10. Together, these data suggest that extreme caution is needed in translating the results of multiplex cytokine profiling into biomarker discovery in psychiatry.

Keywords: Cytokine, Psychiatry, Inflammation, Multiplex immunoassay 


\section{Introduction}

Cytokines belong to a large family of polypeptides that are produced by various cell types in the body, including the cells of the immune system. In particular, pro-inflammatory cytokines such as interleukin (IL)-1 1 , IL-6 and tumor necrosis factor (TNF) are rapidly secreted in response to a pathogen infection, whereas anti-inflammatory cytokines, such as IL-4, IL-10 and IL-13 have the opposite effects. Binding of the cytokines to specific receptors on the cell surfaces triggers events inside the cell that lead to altered gene expression profiles and cell function (Bezbradica and Medzhitov, 2009). In the last two decades, studies have shown that cytokines produced in peripheral tissues can access the central nervous system and interact with the cytokine network in the brain to influence virtually every aspect of brain function relevant to behavior, including neurotransmitter metabolism, neuroendocrine function, synaptic plasticity, and the neurocircuits that regulate mood, feeding, sleep, motor activity, motivation, anxiety and alarm (Capuron et al., 2001; Dantzer et al., 2011; Marshall and Born, 2002; Raison et al., 2006).

During the host immune response to pathogen invasion, the release of proinflammatory cytokines is usually transient and regulated by anti-inflammatory mechanisms. Consequently, the behavioral effects triggered by the increased serum cytokine levels have developped as an adaptive, temporary and controlled reaction of the brain to immune signals (Medzhitov, 2008). Nevertheless, when an immune challenge becomes chronic or unregulated, as occurs in patients who are receiving chronic cytokine treatments or those exposed to chronic medical illness or psychological stress, the cytokines may contribute to the development of clinically relevant behavioral symptoms and neuropsychiatric diseases, including major depression (Raison and Miller, 2013). In support of this hypothesis, depressive symptoms have been shown to be associated with increased serum and plasma concentrations of TNF, IL-6 and C-reactive protein (Dowlati et al., 2010; Goldsmith et al., 2016; Strawbridge et al., 2015; Valkanova et al., 2013). Most importantly, recent longitudinal studies have extended these cross-sectional observations by reporting that increased 
inflammatory markers in non-depressed individuals predict the later development of major depression (Gimeno et al., 2009; Pasco et al., 2010; van den Biggelaar et al., 2007).

Based on these observations, several authors have suggested that specific cytokines could be used as biomarkers in psychiatric diseases (Stuart and Baune, 2014), and even that cytokine-blocking reagents could be used to treat or prevent psychiatric symptoms (Fond et al., 2014; Kohler et al., 2014; Miller and Raison, 2015).

Recently, biomarkers for neuropsychiatric diseases have been classified into those of risk, diagnosis/trait, state, stage, treatment response and prognosis (Davis et al., 2015). In mood disorders, several studies have suggested that the chronic mild inflammation indicated by elevated C-reactive protein (Courtet et al., 2015; Rapaport et al., 2015; Uher et al., 2014) as well as the quantification of the levels of inflammatory cytokines such as IL-6, IL-10 and TNF (de Witte et al., 2014; Ducasse et al., 2015b; Haapakoski et al., 2015; Halaris et al., 2015; Rethorst et al., 2013) could prove to be useful biomarkers for improved diagnosis and the detection of treatment refractoriness (Strawbridge et al., 2015), as well as indicators of the risk of suicide. As an increasing number of inflammatory factors are tested, an emerging picture supports the idea that a complex network of cytokines is involved in the pathophysiology of disease such as major depression (Dahl et al., 2014).

It is known that many blood mediators present substantial individual variations among healthy subjects (Katsuura et al., 2011), and that acute psychological stress as well as acute exercise also produce robust effects on some circulating inflammatory factors in healthy people (Hallberg et al., 2010; Katsuura et al., 2010; Reihmane et al., 2012; Steptoe et al., 2007). In addition, most of the studies in this field have not considered the possibility that inflammatory factors measured in peripheral blood might exhibit a high degree of variability independent of any neuropsychiatric disorder. Some investigators have recognized the risk of making single measurements in healthy controls when assessing the variation due to antidepressant treatment in patients, but they emphasized that the previously obtained measurements were fairly stable, although this has not been documented in the literature (Halaris et al., 2015). Therefore, as a prerequisite for establishing biomarkers, investigators 
should define which factors actually exhibit stable levels in the circulating biofluids over days, weeks, and seasons in healthy adult subjects. Once this is established, it will be possible to distinguish real-time state-related protein concentration changes that are normalized by remission or by antidepressant treatment from the genetically and epigenetically driven physiological or stochastic variations that occur in healthy individuals. Moreover, even the previous results remained only partially reproducible. Although there are several possible explanations for these discrepancies (Zhou et al., 2010), reliability of the tools is one of the first steps required in biomarker development to avoid jumping to premature negative or positive conclusions (Niculescu et al., 2015).

Although the enzyme-linked immunosorbent assay (ELISA) technique has been widely used to measure serum cytokine levels in clinical studies, the recent development of multiplex immunoassays now offers the possibility of simultaneously measuring the levels of expression of large panels of proteins using a small volume of sample and at an affordable price (Altara et al., 2015; Butler et al., 2015; Guest et al., 2011; Halaris et al., 2015; Ho et al., 2015; Janelidze et al., 2013; Nielsen et al., 2015; Schwarz et al., 2012). Nevertheless, it is important for the new and constantly upgraded multiplex technologies to prove that their performance is at least equal to the 'gold standard' ELISA for each analyte tested in the combinations (Bastarache et al., 2014). In addition, the use of complex biological matrices such as sera or plasma must be used to mimic as closely as possible the biological test as it is planned to be used in clinical diagnostics. In biological human fluids, the seasonal, hormonal, and circadian physiological variability in the circulating concentrations of cytokines/chemokines/growth factors remains poorly investigated both in psychiatric patients as a function of their treatments as well as in healthy subjects. Furthermore, large differences exist among individuals (Agorastos et al., 2014; Biancotto et al., 2013; Mueller et al., 2012).

Among the various multiplex platforms, the fluorescent bead-based immunoassay Luminex® (Bio-Rad) and the electro-chemiluminescence immunoassay V-plex® (Meso Scale Discovery) technologies dominate the market (Rosenberg-Hasson et al., 2014). Thus far, very few investigators have compared these technologies and evaluated the limits of 
quantification and intra-assay reproducibility for assay of human blood (Breen et al., 2011; Chowdhury et al., 2009; Fu et al., 2010; Nechansky et al., 2008; Toedter et al., 2008). Therefore, in the context of blood biomarker discovery in psychiatric disorders, we have aimed to evaluate whether the levels of circulating cytokines/chemokines/growth factors that have been identified as candidate biomarkers vary during periods of weeks and months in healthy individuals. For that purpose, we have tested the reliability of the two leading multiplex technologies, i.e. Bio-Plex 200 (Bio-Rad) with Luminex® Cytokine 27-plex assay and QuickPlex (V-PLEX®®) with V-PLEX® Proinflammatory and Cytokine panels (20 markers) using longitudinally collected sera and plasma from both healthy subjects and patients suffering from a major depressive episode (MDE). 


\section{Materials and methods}

\subsection{Populations}

Blood sera and plasma samples were obtained during a 30-week prospective evaluation of a cohort of 10 patients with a MDE according to DSM-IV during a severe depressive episode (Hamilton Depression Rating Scale -17 items (HDRS-17) = 23.3 at inclusion), and 10 sexand age-matched healthy controls. Four visits during the follow-up period (inclusion, 2, 8 and 30 weeks later) allowed for clinical assessment and biological sampling. An extensive clinical and psychological examination, using both Structured Clinical Interview for DSM Disorders (First et al., 2002) and a self-rating questionnaire, as well as clinical and biological examinations (including body temperature, blood cell count) were used to determine the absence of any medical conditions or any psychiatric disorders in the healthy controls. We used the same procedure to exclude any patients with medical comorbidities. None of the subjects had received any vaccinations within a month before the inclusion. Moreover, to confirm the intra-individual stability measured in healthy subjects, 10 additional healthy controls were included. All subjects gave informed written consent. The study was conducted in accordance with the latest version of the Declaration of Helsinki and was approved by the appropriate French ethical and medical authorities. It was part of an ongoing larger study registered in ClinicalTrials.gov with ID: NCT02209142.

\subsection{Blood samples}

Peripheral blood samples were obtained from fasting subjects between 7:00 am and 9:00 am on workdays. Five milliliters of peripheral blood was drawn by venipuncture into both serum and $\mathrm{K}_{2}$ EDTA Vacutainer tubes. For the serum collection, the blood was allowed to clot for 1 hour before centrifugation (1500 x g, $10 \mathrm{~min}$ ). The serum and plasma samples were stored in $0.5 \mathrm{ml}$ aliquots at $-80^{\circ} \mathrm{C}$. For the measurements of the blood cytokine levels, the serum and plasma samples were thawed on ice, and $50-\mu$ laliquots were prepared and stored at $-80^{\circ} \mathrm{C}$. 


\subsection{Bio-Plex immunoassay}

The serum and plasma levels of basic fibroblast growth factor (bFGF), eotaxin (CCL-11), granulocyte colony-stimulating factor (G-CSF), granulocyte-macrophage colony-stimulating factor (GM-CSF), IFN-y, IL-1ß, IL-1RA, IL-2, IL-4, IL-5, IL-6, IL-7, IL-8 (CXCL8), IL-9, IL-10, IL-12p70, IL-13, IL-15, IL-17, IFN-inducible protein-10 (IP-10; CXCL10), monocyte

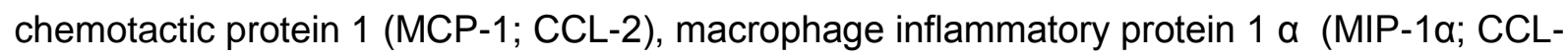
3), MIP-1ß (CCL-4), platelet-derived growth factor BB (PDGF-BB), RANTES (CCL-5), TNF, and vascular endothelial growth factor (VEGF) were analyzed using the Bio-Plex Pro Human Cytokine 27-plex Assay (Bio-Rad) according to the manufacturer's instructions. The serum and plasma samples were diluted 4 -fold and run in duplicate. The samples from the 4 time points of each 10 healthy control subjects and 10 MDE patients were run in a total of 4 plates to measure the serum and plasma cytokine concentrations. Two lots of the reagents were used for the entire study (plate and standards): one for the sera and one for the plasma samples. A minimum of 50 beads per cytokine was acquired. The median fluorescence intensities were collected on a Bio-Plex 200 instrument (Bio-Rad) using Bio-Plex Manager software version 6.0 (Bio-Rad). Standard curves for each cytokine were generated using the premixed lyophilized standards provided in the kits. Serial 4-fold dilutions of the standards were run to generate a 9-standard concentration set, and the diluent alone was used as a blank. The cytokine concentrations in the samples were determined from the standard curve using a 5-parameter logistic fit curve to transform the mean fluorescence intensities into concentrations. The lower limit of quantification (LLOQ) was determined for each cytokine and for each plate as the lowest standard for which both duplicate measurements were above background values (Table 1). The upper limit of quantification (ULOQ) was determined for each cytokine and for each plate as the highest standard that did not reach saturation (Table 1).

\subsection{V-plex® immunoassays}


The serum and plasma levels of IFN-y, IL-1 $\beta$, IL-2, IL-4, IL-6, IL-8 (CXCL8), IL-10, IL-12p70, IL-13, and TNF were measured using the V-plex Proinflammatory Panel 1 (MSD). The serum levels of GM-CSF, IL-1 $\alpha$, IL-5, IL-7, IL-12/IL-23p40, IL-15, IL-16, IL-17A, TNF- $\beta$, and VEGF were measured using the V-plex Cytokine Panel 1 (MSD). All assays were performed according to the manufacturer's instructions. The serum and plasma samples were diluted 2.6-fold. The samples from the 4 time points from each of the 20 healthy controls and 10 MDE patients were run in duplicate. Those from the additional 10 healthy controls were run as single samples. A total of 12 plates were used to measure the serum and plasma cytokine concentrations. Two lots of the reagents were used for the entire study (plate and standards): one for the sera and one for the plasma samples. The data were acquired on the V-plex® Sector Imager 2400 plate reader and analyzed using the Discovery Workbench 3.0 software (MSD). The standard curves for each cytokine were generated using the premixed lyophilized standards provided in the kits. Serial 4-fold dilutions of the standards were run to generate a 7-standard concentration set, and the diluent alone was used as a blank. The cytokine concentrations were determined from the standard curve using a 4-parameter logistic fit curve to transform the mean light intensities into concentrations. The lower limit of quantification (LLOQ) was determined for each cytokine and for each plate as the lowest standard for which both duplicate measurements were above the duplicate background values (Table 1). The upper limit of quantification (ULOQ) was determined for each cytokine and for each plate as the highest standard that did not reach saturation (Table 1).

\subsection{ELISA}

The serum levels of IL-6 were measured using a Quantikine High Sensitivity ELISA kit (R\&D) according to the manufacturer's instructions. Serial 2-fold dilutions of the standard were used to generate a 7-standard concentration set. The diluent was used as a blank. The serum and plasma samples were diluted 2-fold and run separately on a single plate. The data were acquired using an Infinite F200 plate reader (Tecan) and Magellan version 6.5 software 
(Tecan). The IL-6 concentrations were determined from the standard curve using a 4parameter logistic fit curve to transform the mean optical densities into concentrations.

\subsection{Statistical analysis}

A detailed workflow for the analyses is provided in Supplementary Figure 1. The dynamic range of the measurements for each plate corresponded to the values for the concentrations between the LLOQ and the HLOQ. A cytokine was considered detectable with either platform only if at least $60 \%$ of the duplicate measures of the cytokine concentrations lay within the LLOQ-HLOQ window (i.e., the detection range) for the sera from either the patient or control group. The reproducibility of the calibrator dilutions and reproducibility of intra-assay replicates were evaluated by calculating the coefficient of variation (CV) in \% (i.e., CV = standard deviation/mean*100). Since there is no consensus on the appropriate threshold for objective judgment of the reliability of a biological test, we have set up a threshold of $\mathrm{CV}<$ $15 \%$ as revealing good reliability and a CV $<5 \%$ for very good reliability. The measurements of identical cytokines performed on both platforms were compared using Spearman's coefficient of correlation. The intra-individual serum cytokine variability in the healthy subjects was evaluated using the intra-class correlation coefficient (ICC), which reflects the relative balance of between- and within-person variance components (Agalliu et al., 2013; Clendenen et al., 2010; Dossus et al., 2009; Epstein et al., 2013; Gu et al., 2009; Hardikar et al., 2014; Hofmann et al., 2011; Navarro et al., 2012; Wong et al., 2008). As suggested by Rosner (Rosner, 2000), an ICC $\geq 0.75$ indicates excellent reproducibility, $0.4 \leq \rho<0.75$ indicates fair to good reproducibility, and $<0.4$ indicates poor reproducibility. Moreover, an ICC of at least 0.55 has been established as a threshold for cytokines worthy of consideration (Giraudeau, 1996; Gu et al., 2009). All statistical tests were conducted using the IBM SPSS Statistics v22 software. A p-value $<0.05$ was considered as significant. 


\section{Results}

\subsection{Standard curves}

We studied a total of 31 cytokines. Of these, 16 were detected on both platforms, 11 were detected on the Luminex® platform only, and 4 were detected on the V-plex® platform only. The intra-assay variability was good ( $\mathrm{CV}<15 \%)$ for all cytokines on both platforms, and excellent $(\mathrm{CV}<5 \%)$ for 15 (out of 20 ) cytokines on the V-plex® platform and 24 (out of 27 ) on the Luminex® platform (Table 1). When the 16 cytokines that were detected on both platforms were considered, the LLOQ was lower on the V-plex® platform than on the Luminex® platform, except for IL-1ß and IL-13 (Table 1). In contrast, the detection range, measured as the ratio between the HLOQ and the LLOQ, was higher on the Luminex® platform than on the V-PLEX® platform for all cytokines except for IL-2 and IL-15 (Table 1). Nevertheless, when considering the five consecutive four-fold calibrator dilutions that formed the best regression line, the linearity was much closer to $100 \%$ for all tested analytes except for IFN-y and IL-13 on V-PLEX® compared to Luminex® platform as shown in Supplementary Figure 1 and Table 1.

\subsection{Serum and plasma cytokine levels}

Among the 31 proteins that we studied, several were below the LLOQ for more than $60 \%$ of both the tested serum and plasma samples (Tables 2 and 3). This was the case for IL-15 and MCP-1 on the Luminex® platform, IL-4, IL-12p70 and IL-13, and GM-CSF on the V-plex® platform, and IL-2 on both platforms. In general, the comparison of the percentages that were detected in the plasma versus the sera showed that a smaller proportion of the plasma values for most of the analytes were below the LLOQ especially with the Luminex® platform. For example, although IL-15, GM-CSF and IFN-y were undetectable in all sera, they were detected in half, almost all and all of the plasma samples, respectively. Of the 16 cytokines measured by both platforms, only IL-7, IL-8, TNF and VEGF were detected in all or almost sera and plasma samples. 
For the analytes that were detected in more than $60 \%$ of the samples on at least one platform, we measured the intra-assay reproducibility by calculating the $\mathrm{CV}$ for the duplicates. Parallel to the increased detectability in plasma for the Luminex® platform, the intra-assay reproducibility was also better in plasma compared to sera with CV $<15 \%$ observed in 22 assays compared 13 (out of 27 ), respectively. The results from the V-plex® platform were more similar between the sera and plasma, with approximately half of the cytokines demonstrating a CV $<15 \%$. Despite the overall heterogeneity of the performance for both platforms, in examining the assays for which very good reproducibility was obtained, a slight advantage might be given to the $\mathrm{V}$-plex® platform on the basis that more analytes demonstrated CVs that were $<5 \%(30 \%$ for MSD versus $11 \%$ for Luminex for sera and $20 \%$ versus $7 \%$ for plasma).

There is no absolute biological reason that would mandate similar and/or correlated concentration of all analytes in sera and plasma samples from the same individual (Altara et al., 2015; Chaturvedi et al., 2011; Clendenen et al., 2010; Dossus et al., 2009; RosenbergHasson et al., 2014; Wong et al., 2008). We therefore determined on each platform the degree of correlation between serum and plasma measurements. As shown in Table 4 (samples of the first visit only: the results for all four visits are reported on Supplementary Tables 1 and 2), among the analytes that were detected in over $60 \%$ of both sera and plasma with adequate reproducibility of the duplicates, only 5 of 16 (Eotaxin, IP-10, MIP-1 $\beta$, IL-10 and IL-13) were correlated for the two blood fluids in the Luminex® analyses, whereas 7 of 10 (IL-12p40, IL-16, IL-10, IFN-y, IL-6, IL-15, TNF, and VEGF) demonstrated strongly similar of measurements on the MSD platform. Specifically, the correlation of measurements obtained for IL-6 on both multi-assay platforms is shown in Supplementary Fig. 2A and B.

Among the 16 cytokines detected by both immunoassays, only IL-8, IL-10, TNF and VEGF exhibited low intra-assay variability on both platforms for the sera (Table 2 and Supplementary Figure $2 \mathrm{~A}$ and $\mathrm{C}$ ), whereas IFN- $\mathrm{\gamma}$, IL-6, IL-7, IL-8, IL-10, TNF, and VEGF fulfilled these requirements for the plasma (Table 3). For these proteins, we used the Spearman non-parametric correlation test to measure the correlation between the two 
platforms. Table 5 reports the results for the samples from the first visit only, and the results for all four visits are shown in Supplementary Tables 3 and 4 for the serum and plasma samples, respectively. Correlations between the platforms were observed only for IL-8 in serum and for IL-6 in plasma. Moreover, the correlation for IL-6 was not maintained in all visits (Supplementary Table 3). Finally, the correlation was very high in both fluids at all visits only for VEGF.

Furthermore, with the exception of IL-8, the absolute cytokine concentrations measured by the two platforms were substantially different and exceeded 10-fold for IL-6, IL10, IL-17 and TNF and 200-fold for IL-12p70 in plasma and/or sera (Supplementary Table 5).

Because several studies have suggested to a critical role for IL-6 in MDE, we specifically investigated whether the levels of IL-6 measured by multiplex assays were comparable to those measured by ELISA. In the serum samples, while no correlation was observed for all visits between Luminex $\AA$ and ELISA platforms (Fig. 1A), the correlation was very robust between the $M S D$ and ELISA (visit 1 , Rho=0.781, $p=4.86 E-5$; Fig. 1B). In plasma, again, the correlation never reached significance for Luminex® (Fig. 1C) in contrast to the MSD (Fig. 1D). As a control, we also verified the high correlation between all serum and plasma sample measurements for ELISA (visit 1, Rho=0.826, $p=7.12 \mathrm{E}-6$; Supplementary Fig. 2C).

\subsection{Intra-individual variability in healthy subjects}

As a prerequisite for the possible use of the 31 analytes that we have assayed as trait or state biomarkers in psychiatric diseases, we investigated whether their levels were stable over time in healthy individuals. Therefore, we calculated the ICC (Table 6). For some analytes, we could confirm intra-individual stability during a 30-week follow-up in healthy subjects on the basis of the convergent results in serum and plasma or between platforms (Eotaxin, bFGF, G-CSF, GM-CSF, IL-1 $\beta$, IL-4, IL-5, IL-7, IL-9, IL-12p40, IL-12p70, IL-15, MIP-1 $\alpha$, MIP-1 $\beta$, RANTES, and TNF), whereas for others, intra-individual variability overtime in healthy subjects is most likely the rule (IL-8, IL-10, IP-10). As an example, the discrepancy 
between the very low intra-individual stability of IL-8 across time in both serum and plasma and the strong stability of TNF is shown on Supplementary Fig. 3. Additionally, for some analytes, the results were discordant according to either the biofluid analyzed (i.e., serum versus plasma) or the platform used to assay the analyte (IFN-y, IL-6, IL-10, IL-13, IL-16, IL17, PDGF-BB, VEGF). As an illustration, the poor to intermediate stability of both serum and plasma IL-6 measured on the MSD platform is represented on Fig. 1E and 1F. To confirm the level of stability for some of the most studied cytokines in the field of neuropsychiatry, we determined the ICC from serum and plasma samples of 10 additional healthy subjects during the 30 week-follow-up period assayed with the MSD platform. As shown in Supplementary Table 6, except for TNF- $\beta$, which was ultimately found to be stable in sera, we confirmed all the other trends described above. 
R. Belzeaux et al.

\section{Discussion}

To our knowledge, this is the first investigation of the stability of protein biomarker candidates in healthy individuals over time based on multiplex cytokine profiling. We also measured these target proteins in both sera and plasma from patients with a psychiatric disorder because these subjects present specific biological characteristics comparable with other infectious or pure inflammatory diseases. Meta-analyses that included both sera and plasma samples have previously identified only small to moderate effects on the cytokine levels within psychiatric populations (Dowlati et al., 2010; Ducasse et al., 2015a; Hiles et al., 2012; Valkanova et al., 2013). As a consequence, there is a great need in immuno-psychiatric studies for tools that may have the abilities to detect such low levels and small mean differences.

Not much is known about physiological variations of circulating inflammatory signaling proteins in healthy individuals over weeks, months or even years. Nevertheless, contrary to the intuitive idea that many biological processes are not prone to variability outside of pathological situations, we have found that the concentrations of at least a few circulating factors exhibited intra-individual variability in healthy subjects. This was the case for IFN- $\gamma$, IL-6, IL-8, IL-10 and IP-10. In the case of IL-8, a similar concern was raised in previous investigations (Epstein et al., 2013; Gu et al., 2009; Hofmann et al., 2011). Therefore, when comparing the values of these cytokines in patients with control subjects, a single time point is not adequate to evaluate these cytokines as potential biomarkers.

We have also confirmed early reports that showed discrepancies between the technical precision of multiplex technologies using calibrator samples and the heterogeneous platform assay performance with complex human body fluids (Nechansky et al., 2008; Toedter et al., 2008). On the one hand, our results confirmed both manufacturer's information and previous work based on lyophilized cytokine standards (Chowdhury et al., 2009). On the other hand, unexpectedly, dissimilar results and heterogeneous performances were obtained for human sera and plasma. 
Neither platform demonstrated any clear advantage over the other one tested, but some trends were observed. The sensitivity was generally higher for the V-PLEX® platform, compared to Luminex® platform, as previously reported (Breen et al., 2011; Chowdhury et al., 2009). Despite the large concentration range obtained for the Luminex® standard curves, the slope steepness was higher with V-PLEX® for the low concentrations, and a better conservation of linearity was achieved (Supplementary Figure 1, Table 1). These results reflected a better capacity to detect low-abundance cytokines. For some assays, the proteins could not be detected in more than $60 \%$ of the tested sera (Table 2 ). Indeed, both platforms failed to robustly detect the GM-CSF, IL-1 $\beta$, IL-2 and IL-5 cytokines for which previous investigations had reported significant variations in expression either during MDE treatment or at baseline in comparison to healthy controls (Biancotto et al., 2013; Dahl et al., 2014; Hernandez et al., 2008; Schmidt et al., 2014; Shelton et al., 2015). These types of inconsistency in the results have already been reported (Breen et al., 2011; Chaturvedi et al., 2011; Dabitao et al., 2011; Dupuy et al., 2013; Fu et al., 2010). In particular, the consensus that the detection of IL-1 $\beta$ suffers from poor performances is of importance. An inflammatory cytokine could be a sensitive biomarker for a depressive mood even in healthy subjects (Katsuura et al., 2011). It is also possible that such a cytokine could contribute to the depressive symptoms, and its use as a biomarker could even reveal how antidepressants block the effects of inflammation on the brain (Hannestad et al., 2011; Hernandez et al., 2013). However, a lack of reliability in the measurements weakens the use of multiplex assays for clinical purposes. Moreover, using the same Bio-Plex cytokine reagent kit used in a previous study on healthy university students, we also confirmed the lack of detection of bFGF, IL-15 and MCP-1 in serum (Katsuura et al., 2010; Katsuura et al., 2011).

The intra-assay reproductibility was heterogeneous among the cytokines and between the platforms (Tables 2 and 3). In contrast, the performance of both platforms was sufficiently consistent for IL-8, IL-10, TNF and VEGF, although there was a general advantage for V-PLEX® technology. Importantly, these factors have shown promise as biomarkers for MDE (Carvalho et al., 2015; Meyer et al., 2011; Young et al., 2014). 
It has already been stressed out that the comparison of absolute cytokine concentration should be attempted only if used reagents were obtained from the same supplier (Khan et al., 2004), although even singleplex assays compared to multiplex assays from the same company can yield significantly different results (de Koning et al., 2012; Huttunen et al., 2014). Indeed, we confirmed that the absolute values of the proteins detected were different between the two tested technologies. Such discordance could be due to differences in the capture and/or detection antibodies and to a differential sensitivity to matrix interference (Dupuy et al., 2013; Khan et al., 2004). However, among the cytokines for which the quantification was consistent, we observed strong inter-platform correlations for IL8 and VEGF (Table 5), in accordance with previous findings (Chowdhury et al., 2009; Dabitao et al., 2011; Huttunen et al., 2014). We also confirmed the tight relationship between V-PLEX® and ELISA measurements for IL-6 (Bastarache et al., 2014; Huttunen et al., 2014).

Very few studies have compared the serum and plasma concentrations of cytokines from the same individuals (Altara et al., 2015; Chaturvedi et al., 2011; Clendenen et al., 2010; Dossus et al., 2009; Rosenberg-Hasson et al., 2014; Wong et al., 2008). In general, it has been found that these marker measurements are not interchangeable between serum and plasma. Consistent with these previous studies, we found that in most cases, the serum and plasma concentrations of the cytokines were not correlated (Table 4). Therefore, metaanalyses should distinguish studies of serum samples from the ones that used plasma samples. Moreover, some investigators have reported that the plasma cytokine concentrations were generally higher than those measured in serum due to a higher nonspecific background observed in serum (Chaturvedi et al., 2011; Rosenberg-Hasson et al., 2014; Wong et al., 2008). In our study, we have confirmed such a trend only for the Luminex® platform (Supplementary Table 5), which may explain why the detectability was better for that platform in plasma compared to sera (Tables 2 and 3).

Our findings have several important technical and theoretical limitations. First, we could not compare exactly the same sets of cytokines on both platforms on the basis of the commercial availability, and it has previously been observed that assay performance 
decreases with increasing numbers of markers on a panel (Chaturvedi et al., 2011). It will be important in the future to follow up with studies to compare larger sets of cytokines as similar to each other as possible, because recent investigations in the field of biomarker discovery have revealed new candidates for promising proteins. Secondly, we did not address the inter-assay variability on each platform, which is an important factor to consider especially when investigating large samples that cannot be processed with a single batch of reagents or for longitudinal studies when assaying the samples at different days over long periods of time. Third, our results should be considered to be preliminary on the basis of the relatively small sample size that makes any group comparison between healthy control and patients inappropriate. Of note, for the purpose of another study, all serum samples assayed in the present study, and obtained from healthy control and MDE patients at their first visit, have been tested again on the MSD platform with a different batch of reagents and a two-year interval (data not shown). The same panels of cytokines were assayed as well as an additional chemokine panel. A comparative analysis of old and new measurements of cytokine concentrations revealed robust correlations for IFN- $y$, IL-6, IL-8, IL-10, IL-12p40, IL15, IL-16, TNF, TNF- $\beta$, and VEGF (data not shown), suggesting a strong inter-assay stability for these analytes on the MSD platform. Moreover, the new measurements for IP-10 and MIP-1 $\beta$ obtained on the MSD platform strongly correlated with previous measurements obtained with the Luminex platform (data not shown), suggesting that these assays exhibit low intra- and inter-assay variability on both platforms.

When possible, we followed the manufacturer's recommendations, and therefore used a different dilution factor for both the sera and plasma samples for the Bio-Plex (factor 4) compared to the V-PLEX (factor 2.6) assays. This could explain, at least in part, the increased sensitivity of the latter. Nevertheless, it has been shown that (i) both serum and plasma inhibit the readouts, (ii) the inhibitory effect of the matrix is partly reversed by dilution, and (iii) this type of inhibition is much higher with the Luminex® system compared to the MSD assays (Rosenberg-Hasson et al., 2014). In addition, as previously noted in a few studies (Dossus et al., 2009; Dupuy et al., 2013; Toedter et al., 2008), the nature of the 
capture/detection antibody reactivity is crucial. In fact, for some cytokines such as IL-4, IL12 p70 and IL-17 the Bio-Plex assays invariably outperformed those of the V-PLEX in both sera and plasma, whereas for other analytes including bFGF, GM-CSF and IL-15, striking differences in the detectability between sera and plasma were observed.

The detection range, measured as the ratio between the HLOQ and the LLOQ was over 1000 for all tested analytes on both platforms (Table 1). However, the relevant parts of the calibration curves that were used to determine the protein concentrations generally included only 2 points or less in the lower end of the curve. Therefore, for at least the key cytokines, the availability of ultrasensitive multiplex assays dedicated to the very low end of calibration curves would be a significant advance as some investigators began to set-up (Rivnak et al., 2015). A dynamic range of $0-20 \mathrm{pg} / \mathrm{ml}$ would be optimal. In addition, the high variability in the measurement may result from factors other than those driven by the technology. The observed differences between the platforms were unlikely to have resulted from variability within the samples themselves because we ensured that identical aliquots were used on both platforms, avoided extra cycles of freezing/thawing procedures, performed the multisite measurements within the same period, and limited the manipulations to an experienced operator. Nevertheless, a recent large multi-site study reported highly variable results despite assessment of identical samples with same batches of reagents and identical Luminex-based assays (Lynch et al., 2014). Therefore, we cannot totally exclude the operator on each platform as a source of the variability.

Many non-pathological processes can transiently affect the concentration of cytokines, chemokines and growth factors. For example, diurnal rhythms have been reported to produce significant differences between serum and plasma (Altara et al., 2015; Chaturvedi et al., 2011; Clendenen et al., 2010; Rosenberg-Hasson et al., 2014; Wong et al., 2008). For this reason, all of the subjects in our control and patient cohorts were examined under the same fasting conditions in the morning. Nevertheless, more labile factors could alter the cytokine concentrations within the few minutes prior to the blood collection. Among those factors, brief natural stresses in healthy individuals have been shown to affect circulating 
cytokine levels (Katsuura et al., 2010). It is thus possible that some of the fluctuations that we observed were in fact driven by some physiological stress reaction that need to be further demonstrated.

As a consequence, we feel that these results could underscore the necessity to perform assays in duplicates when using such multiplex platforms and to provide clear information regarding the intra-assay reliability within samples. Moreover, to avoid premature positive or negative conclusions, attention needs to be paid to the intra-individual variability. Longitudinal follow-up studies with repetitive measures may be a solution to overcome these issues. However, we cannot rule out the possibility that for some cytokines, the stochastic or cyclic variations in protein concentrations measured in healthy adults may stabilize with disease, in which case the stability could become a marker for the pathological condition. Finally, even after controlling for all previously defined potential biases, cross validation using another method, such as ELISA, may also be necessary (Leng et al., 2008; Zhou et al., 2010), as demonstrated in our data by the potential lack of correlation between platforms for TNF and IL-10 (Table 5). 
R. Belzeaux et al.

\section{Conclusions}

Although they have received considerable interest for exploratory studies in the field of psychiatry and behavioral sciences, multiplex platforms have to be considered with caution. Any preliminary results would have to be interpreted rigorously according to current performance limits and would obviously require validation by standard ELISA methods. In addition, the issue of circulating protein stability in normal healthy subjects is not trivial. Any candidate biomarker for a neuropsychiatric disease would need to be validated over repeated measurements in healthy controls a low level of variability. 
R. Belzeaux et al.

\section{References}

Agalliu, I., Xue, X., Cushman, M., Cornell, E., Hsing, A.W., Kaplan, R.C., Anastos, K., Rajpathak, S., Ho, G.Y., 2013. Detectability and reproducibility of plasma levels of chemokines and soluble receptors. Results Immunol 3, 79-84.

Agorastos, A., Hauger, R.L., Barkauskas, D.A., Moeller-Bertram, T., Clopton, P.L., Haji, U., Lohr, J.B., Geracioti, T.D., Jr., Patel, P.M., Chrousos, G.P., Baker, D.G., 2014. Circadian rhythmicity, variability and correlation of interleukin-6 levels in plasma and cerebrospinal fluid of healthy men. Psychoneuroendocrinology 44, 71-82.

Altara, R., Manca, M., Hermans, K.C., Daskalopoulos, E.P., Brunner-La Rocca, H.P., Hermans, R.J., Struijker-Boudier, H.A., Blankesteijn, M.W., 2015. Diurnal rhythms of serum and plasma cytokine profiles in healthy elderly individuals assessed using membrane based multiplexed immunoassay. J Transl Med 13, 129.

Bastarache, J.A., Koyama, T., Wickersham, N.E., Ware, L.B., 2014. Validation of a multiplex electrochemiluminescent immunoassay platform in human and mouse samples. J Immunol Methods 408, 13-23.

Bezbradica, J.S., Medzhitov, R., 2009. Integration of cytokine and heterologous receptor signaling pathways. Nat Immunol 10, 333-339.

Biancotto, A., Wank, A., Perl, S., Cook, W., Olnes, M.J., Dagur, P.K., Fuchs, J.C., Langweiler, M., Wang, E., McCoy, J.P., 2013. Baseline levels and temporal stability of 27 multiplexed serum cytokine concentrations in healthy subjects. PLoS One 8, e76091.

Breen, E.C., Reynolds, S.M., Cox, C., Jacobson, L.P., Magpantay, L., Mulder, C.B., Dibben, O., Margolick, J.B., Bream, J.H., Sambrano, E., Martinez-Maza, O., Sinclair, E., Borrow, P., Landay, A.L., Rinaldo, C.R., Norris, P.J., 2011. Multisite comparison of high-sensitivity multiplex cytokine assays. Clin Vaccine Immunol 18, 1229-1242.

Butler, M.G., Hossain, W., Sulsona, C., Driscoll, D.J., Manzardo, A.M., 2015. Increased plasma chemokine levels in children with Prader-Willi syndrome. Am J Med Genet A 167A, 563-571. 
Capuron, L., Bluthe, R.M., Dantzer, R., 2001. Cytokines in clinical psychiatry. Am J Psychiatry 158, 1163-1164.

Carvalho, A.F., Kohler, C.A., Mclntyre, R.S., Knochel, C., Brunoni, A.R., Thase, M.E., Quevedo, J., Fernandes, B.S., Berk, M., 2015. Peripheral vascular endothelial growth factor as a novel depression biomarker: A meta-analysis. Psychoneuroendocrinology 62, 18-26.

Chaturvedi, A.K., Kemp, T.J., Pfeiffer, R.M., Biancotto, A., Williams, M., Munuo, S., Purdue, M.P., Hsing, A.W., Pinto, L., McCoy, J.P., Hildesheim, A., 2011. Evaluation of multiplexed cytokine and inflammation marker measurements: a methodologic study. Cancer Epidemiol Biomarkers Prev 20, 1902-1911.

Chowdhury, F., Williams, A., Johnson, P., 2009. Validation and comparison of two multiplex technologies, Luminex ${ }^{\circledR}$ and Mesoscale Discovery, for human cytokine profiling. J Immunol Methods 340, 55-64.

Clendenen, T.V., Arslan, A.A., Lokshin, A.E., Idahl, A., Hallmans, G., Koenig, K.L., Marrangoni, A.M., Nolen, B.M., Ohlson, N., Zeleniuch-Jacquotte, A., Lundin, E., 2010. Temporal reliability of cytokines and growth factors in EDTA plasma. BMC Res Notes 3, 302.

Courtet, P., Jaussent, I., Genty, C., Dupuy, A.M., Guillaume, S., Ducasse, D., Olie, E., 2015. Increased CRP levels may be a trait marker of suicidal attempt. Eur Neuropsychopharmacol.

Dabitao, D., Margolick, J.B., Lopez, J., Bream, J.H., 2011. Multiplex measurement of proinflammatory cytokines in human serum: comparison of the Meso Scale Discovery electrochemiluminescence assay and the Cytometric Bead Array. J Immunol Methods 372, 71-77.

Dahl, J., Ormstad, H., Aass, H.C., Malt, U.F., Bendz, L.T., Sandvik, L., Brundin, L., Andreassen, O.A., 2014. The plasma levels of various cytokines are increased during ongoing depression and are reduced to normal levels after recovery. Psychoneuroendocrinology 45, 77-86.

Dantzer, R., O'Connor, J.C., Lawson, M.A., Kelley, K.W., 2011. Inflammation-associated depression: from serotonin to kynurenine. Psychoneuroendocrinology 36, 426-436. 
Davis, J., Maes, M., Andreazza, A., McGrath, J.J., Tye, S.J., Berk, M., 2015. Towards a classification of biomarkers of neuropsychiatric disease: from encompass to compass. Mol Psychiatry 20, 152-153. de Koning, L., Liptak, C., Shkreta, A., Bradwin, G., Hu, F.B., Pradhan, A.D., Rifai, N., Kellogg, M.D., 2012. A multiplex immunoassay gives different results than singleplex immunoassays which may bias epidemiologic associations. Clin Biochem 45, 848-851.

de Witte, L., Tomasik, J., Schwarz, E., Guest, P.C., Rahmoune, H., Kahn, R.S., Bahn, S., 2014. Cytokine alterations in first-episode schizophrenia patients before and after antipsychotic treatment. Schizophr Res 154, 23-29.

Dossus, L., Becker, S., Achaintre, D., Kaaks, R., Rinaldi, S., 2009. Validity of multiplex-based assays for cytokine measurements in serum and plasma from "non-diseased" subjects: comparison with ELISA. J Immunol Methods 350, 125-132.

Dowlati, Y., Herrmann, N., Swardfager, W., Liu, H., Sham, L., Reim, E.K., Lanctot, K.L., 2010. A metaanalysis of cytokines in major depression. Biol Psychiatry 67, 446-457.

Ducasse, D., Jaussent, I., Guillaume, S., Azorin, J.M., Bellivier, F., Belzeaux, R., Bougerol, T., Etain, B., Gard, S., Henry, C., Kahn, J.P., Leboyer, M., Loftus, J., Passerieux, C., Courtet, P.H., Olie, E., 2015a. Increased risk of suicide attempt in bipolar patients with severe tobacco dependence. J Affect Disord 183, 113-118.

Ducasse, D., Olie, E., Guillaume, S., Artero, S., Courtet, P., 2015b. A meta-analysis of cytokines in suicidal behavior. Brain Behav Immun 46, 203-211.

Dupuy, A.M., Kuster, N., Lizard, G., Ragot, K., Lehmann, S., Gallix, B., Cristol, J.P., 2013. Performance evaluation of human cytokines profiles obtained by various multiplexed-based technologies underlines a need for standardization. Clin Chem Lab Med 51, 1385-1393.

Epstein, M.M., Breen, E.C., Magpantay, L., Detels, R., Lepone, L., Penugonda, S., Bream, J.H., Jacobson, L.P., Martinez-Maza, O., Birmann, B.M., 2013. Temporal stability of serum concentrations of cytokines and soluble receptors measured across two years in low-risk HIVseronegative men. Cancer Epidemiol Biomarkers Prev 22, 2009-2015. 
First, M.B., Spitzer, R.L., Gibbon, M., Williams, J.B.W., 2002. Structured Clinical Interview for DSM-IVTR Axis I Disorders, Research Version, Patient Edition. (SCID-I/P). New York State Psychiatric Institute, New York.

Fond, G., Hamdani, N., Kapczinski, F., Boukouaci, W., Drancourt, N., Dargel, A., Oliveira, J., Le Guen, E., Marlinge, E., Tamouza, R., Leboyer, M., 2014. Effectiveness and tolerance of anti-inflammatory drugs' add-on therapy in major mental disorders: a systematic qualitative review. Acta Psychiatr Scand 129, 163-179.

Fu, Q., Zhu, J., Van Eyk, J.E., 2010. Comparison of multiplex immunoassay platforms. Clin Chem 56, 314-318.

Gimeno, D., Kivimaki, M., Brunner, E.J., Elovainio, M., De Vogli, R., Steptoe, A., Kumari, M., Lowe, G.D., Rumley, A., Marmot, M.G., Ferrie, J.E., 2009. Associations of C-reactive protein and interleukin-6 with cognitive symptoms of depression: 12-year follow-up of the Whitehall II study. Psychol Med 39, 413-423.

Giraudeau, B., 1996. Negative values of the intraclass correlation coefficient are not theoretically possible. J Clin Epidemiol 49, 1205-1206.

Goldsmith, D.R., Rapaport, M.H., Miller, B.J., 2016. A meta-analysis of blood cytokine network alterations in psychiatric patients: comparisons between schizophrenia, bipolar disorder and depression. Mol Psychiatry.

Gu, Y., Zeleniuch-Jacquotte, A., Linkov, F., Koenig, K.L., Liu, M., Velikokhatnaya, L., Shore, R.E., Marrangoni, A., Toniolo, P., Lokshin, A.E., Arslan, A.A., 2009. Reproducibility of serum cytokines and growth factors. Cytokine 45, 44-49.

Guest, P.C., Schwarz, E., Krishnamurthy, D., Harris, L.W., Leweke, F.M., Rothermundt, M., van Beveren, N.J., Spain, M., Barnes, A., Steiner, J., Rahmoune, H., Bahn, S., 2011. Altered levels of circulating insulin and other neuroendocrine hormones associated with the onset of schizophrenia. Psychoneuroendocrinology 36, 1092-1096. 
Haapakoski, R., Mathieu, J., Ebmeier, K.P., Alenius, H., Kivimaki, M., 2015. Cumulative meta-analysis of interleukins 6 and 1beta, tumour necrosis factor alpha and C-reactive protein in patients with major depressive disorder. Brain Behav Immun 49, 206-215.

Halaris, A., Myint, A.M., Savant, V., Meresh, E., Lim, E., Guillemin, G., Hoppensteadt, D., Fareed, J., Sinacore, J., 2015. Does escitalopram reduce neurotoxicity in major depression? J Psychiatr Res $66-67,118-126$.

Hallberg, L., Janelidze, S., Engstrom, G., Wisen, A.G., Westrin, A., Brundin, L., 2010. Exercise-induced release of cytokines in patients with major depressive disorder. J Affect Disord 126, 262-267.

Hannestad, J., DellaGioia, N., Bloch, M., 2011. The effect of antidepressant medication treatment on serum levels of inflammatory cytokines: a meta-analysis. Neuropsychopharmacology 36, 24522459.

Hardikar, S., Song, X., Kratz, M., Anderson, G.L., Blount, P.L., Reid, B.J., Vaughan, T.L., White, E., 2014. Intraindividual variability over time in plasma biomarkers of inflammation and effects of longterm storage. Cancer Causes Control 25, 969-976.

Hernandez, M.E., Mendieta, D., Martinez-Fong, D., Loria, F., Moreno, J., Estrada, I., Bojalil, R., Pavon, L., 2008. Variations in circulating cytokine levels during 52 week course of treatment with SSRI for major depressive disorder. Eur Neuropsychopharmacol 18, 917-924.

Hernandez, M.E., Mendieta, D., Perez-Tapia, M., Bojalil, R., Estrada-Garcia, I., Estrada-Parra, S., Pavon, L., 2013. Effect of selective serotonin reuptake inhibitors and immunomodulator on cytokines levels: an alternative therapy for patients with major depressive disorder. Clin Dev Immunol 2013, 267871.

Hiles, S.A., Baker, A.L., de Malmanche, T., Attia, J., 2012. A meta-analysis of differences in IL-6 and IL10 between people with and without depression: exploring the causes of heterogeneity. Brain Behav Immun 26, 1180-1188. 
Ho, P.S., Yeh, Y.W., Huang, S.Y., Liang, C.S., 2015. A shift toward T helper 2 responses and an increase in modulators of innate immunity in depressed patients treated with escitalopram. Psychoneuroendocrinology 53, 246-255.

Hofmann, J.N., Yu, K., Bagni, R.K., Lan, Q., Rothman, N., Purdue, M.P., 2011. Intra-individual variability over time in serum cytokine levels among participants in the prostate, lung, colorectal, and ovarian cancer screening Trial. Cytokine 56, 145-148.

Huttunen, K., Tiihonen, K., Roponen, M., Heederik, D., Zock, J.P., Taubel, M., Hyvarinen, A., Hirvonen, M.R., 2014. The effect of assay type and sample matrix on detected cytokine concentrations in human blood serum and nasal lavage fluid. J Pharm Biomed Anal 96, 151-155.

Janelidze, S., Ventorp, F., Erhardt, S., Hansson, O., Minthon, L., Flax, J., Samuelsson, M., TraskmanBendz, L., Brundin, L., 2013. Altered chemokine levels in the cerebrospinal fluid and plasma of suicide attempters. Psychoneuroendocrinology 38, 853-862.

Katsuura, S., Kamezaki, Y., Tominaga, K., Masuda, K., Nishida, K., Yamamoto, Y., Takeo, K., Yamagishi, N., Tanahashi, T., Kawai, T., Rokutan, K., 2010. High-throughput screening of brief naturalistic stress-responsive cytokines in university students taking examinations. Int J Psychophysiol 77, 135-140.

Katsuura, S., Kamezaki, Y., Yamagishi, N., Kuwano, Y., Nishida, K., Masuda, K., Tanahashi, T., Kawai, T., Arisawa, K., Rokutan, K., 2011. Circulating vascular endothelial growth factor is independently and negatively associated with trait anxiety and depressive mood in healthy Japanese university students. Int J Psychophysiol 81, 38-43.

Khan, S.S., Smith, M.S., Reda, D., Suffredini, A.F., McCoy, J.P., Jr., 2004. Multiplex bead array assays for detection of soluble cytokines: comparisons of sensitivity and quantitative values among kits from multiple manufacturers. Cytometry B Clin Cytom 61, 35-39.

Kohler, O., Benros, M.E., Nordentoft, M., Farkouh, M.E., Iyengar, R.L., Mors, O., Krogh, J., 2014. Effect of anti-inflammatory treatment on depression, depressive symptoms, and adverse effects: a systematic review and meta-analysis of randomized clinical trials. JAMA Psychiatry 71, 1381-1391. 
Leng, S.X., McElhaney, J.E., Walston, J.D., Xie, D., Fedarko, N.S., Kuchel, G.A., 2008. ELISA and multiplex technologies for cytokine measurement in inflammation and aging research. J Gerontol A Biol Sci Med Sci 63, 879-884.

Lynch, H.E., Sanchez, A.M., D'Souza, M.P., Rountree, W., Denny, T.N., Kalos, M., Sempowski, G.D., 2014. Development and implementation of a proficiency testing program for Luminex ${ }^{\circledR}$ beadbased cytokine assays. J Immunol Methods 409, 62-71.

Marshall, L., Born, J., 2002. Brain-immune interactions in sleep. Int Rev Neurobiol 52, 93-131.

Medzhitov, R., 2008. Origin and physiological roles of inflammation. Nature 454, 428-435.

Meyer, T., Stanske, B., Kochen, M.M., Cordes, A., Yuksel, I., Wachter, R., Luers, C., Scherer, M., Binder, L., Pieske, B., Herrmann-Lingen, C., 2011. Serum levels of interleukin-6 and interleukin-10 in relation to depression scores in patients with cardiovascular risk factors. Behav Med 37, 105112.

Miller, A.H., Raison, C.L., 2015. Are Anti-inflammatory Therapies Viable Treatments for Psychiatric Disorders?: Where the Rubber Meets the Road. JAMA Psychiatry 72, 527-528.

Mueller, S.C., Marz, R., Schmolz, M., Drewelow, B., 2012. Intraindividual long term stability and response corridors of cytokines in healthy volunteers detected by a standardized whole-blood culture system for bed-side application. BMC Med Res Methodol 12, 112.

Navarro, S.L., Brasky, T.M., Schwarz, Y., Song, X., Wang, C.Y., Kristal, A.R., Kratz, M., White, E., Lampe, J.W., 2012. Reliability of serum biomarkers of inflammation from repeated measures in healthy individuals. Cancer Epidemiol Biomarkers Prev 21, 1167-1170.

Nechansky, A., Grunt, S., Roitt, I.M., Kircheis, R., 2008. Comparison of the Calibration Standards of Three Commercially Available Multiplex Kits for Human Cytokine Measurement to WHO Standards Reveals Striking Differences. Biomark Insights 3, 227-235.

Niculescu, A.B., Levey, D., Le-Niculescu, H., Niculescu, E., Kurian, S.M., Salomon, D., 2015. Psychiatric blood biomarkers: avoiding jumping to premature negative or positive conclusions. Mol Psychiatry 20, 286-288. 
Nielsen, P.R., Agerbo, E., Skogstrand, K., Hougaard, D.M., Meyer, U., Mortensen, P.B., 2015. Neonatal levels of inflammatory markers and later risk of schizophrenia. Biol Psychiatry 77, 548-555.

Pasco, J.A., Nicholson, G.C., Williams, L.J., Jacka, F.N., Henry, M.J., Kotowicz, M.A., Schneider, H.G., Leonard, B.E., Berk, M., 2010. Association of high-sensitivity C-reactive protein with de novo major depression. Br J Psychiatry 197, 372-377.

Raison, C.L., Capuron, L., Miller, A.H., 2006. Cytokines sing the blues: inflammation and the pathogenesis of depression. Trends Immunol 27, 24-31.

Raison, C.L., Miller, A.H., 2013. Role of inflammation in depression: implications for phenomenology, pathophysiology and treatment. Mod Trends Pharmacopsychiatri 28, 33-48.

Rapaport, M.H., Nierenberg, A.A., Schettler, P.J., Kinkead, B., Cardoos, A., Walker, R., Mischoulon, D., 2015. Inflammation as a predictive biomarker for response to omega-3 fatty acids in major depressive disorder: a proof-of-concept study. Mol Psychiatry.

Reihmane, D., Jurka, A., Tretjakovs, P., 2012. The relationship between maximal exercise-induced increases in serum IL-6, MPO and MMP-9 concentrations. Scand J Immunol 76, 188-192.

Rethorst, C.D., Toups, M.S., Greer, T.L., Nakonezny, P.A., Carmody, T.J., Grannemann, B.D., Huebinger, R.M., Barber, R.C., Trivedi, M.H., 2013. Pro-inflammatory cytokines as predictors of antidepressant effects of exercise in major depressive disorder. Mol Psychiatry 18, 1119-1124.

Rivnak, A.J., Rissin, D.M., Kan, C.W., Song, L., Fishburn, M.W., Piech, T., Campbell, T.G., DuPont, D.R., Gardel, M., Sullivan, S., Pink, B.A., Cabrera, C.G., Fournier, D.R., Duffy, D.C., 2015. A fullyautomated, six-plex single molecule immunoassay for measuring cytokines in blood. J Immunol Methods 424, 20-27.

Rosenberg-Hasson, Y., Hansmann, L., Liedtke, M., Herschmann, I., Maecker, H.T., 2014. Effects of serum and plasma matrices on multiplex immunoassays. Immunol Res 58, 224-233.

Rosner, B., 2000. Fundamentals of Biostatistics, 5th ed. ed. Duxbury Press, Pacific Grove, CA.

Schmidt, F.M., Lichtblau, N., Minkwitz, J., Chittka, T., Thormann, J., Kirkby, K.C., Sander, C., Mergl, R., Fasshauer, M., Stumvoll, M., Holdt, L.M., Teupser, D., Hegerl, U., Himmerich, H., 2014. Cytokine 
levels in depressed and non-depressed subjects, and masking effects of obesity. J Psychiatr Res $55,29-34$.

Schwarz, E., Guest, P.C., Rahmoune, H., Harris, L.W., Wang, L., Leweke, F.M., Rothermundt, M., Bogerts, B., Koethe, D., Kranaster, L., Ohrmann, P., Suslow, T., McAllister, G., Spain, M., Barnes, A., van Beveren, N.J., Baron-Cohen, S., Steiner, J., Torrey, F.E., Yolken, R.H., Bahn, S., 2012. Identification of a biological signature for schizophrenia in serum. Mol Psychiatry 17, 494-502.

Shelton, R.C., Falola, M., Li, L., Zajecka, J., Fava, M., Papakostas, G.I., 2015. The pro-inflammatory profile of depressed patients is (partly) related to obesity. J Psychiatr Res 70, 91-97.

Steptoe, A., Hamer, M., Chida, Y., 2007. The effects of acute psychological stress on circulating inflammatory factors in humans: a review and meta-analysis. Brain Behav Immun 21, 901-912.

Strawbridge, R., Arnone, D., Danese, A., Papadopoulos, A., Herane Vives, A., Cleare, A.J., 2015. Inflammation and clinical response to treatment in depression: A meta-analysis. Eur Neuropsychopharmacol 25, 1532-1543.

Stuart, M.J., Baune, B.T., 2014. Chemokines and chemokine receptors in mood disorders, schizophrenia, and cognitive impairment: a systematic review of biomarker studies. Neurosci Biobehav Rev 42, 93-115.

Toedter, G., Hayden, K., Wagner, C., Brodmerkel, C., 2008. Simultaneous detection of eight analytes in human serum by two commercially available platforms for multiplex cytokine analysis. Clin Vaccine Immunol 15, 42-48.

Uher, R., Tansey, K.E., Dew, T., Maier, W., Mors, O., Hauser, J., Dernovsek, M.Z., Henigsberg, N., Souery, D., Farmer, A., McGuffin, P., 2014. An inflammatory biomarker as a differential predictor of outcome of depression treatment with escitalopram and nortriptyline. Am J Psychiatry 171, $1278-1286$.

Valkanova, V., Ebmeier, K.P., Allan, C.L., 2013. CRP, IL-6 and depression: a systematic review and meta-analysis of longitudinal studies. J Affect Disord 150, 736-744. 
van den Biggelaar, A.H., Gussekloo, J., de Craen, A.J., Frolich, M., Stek, M.L., van der Mast, R.C., Westendorp, R.G., 2007. Inflammation and interleukin-1 signaling network contribute to depressive symptoms but not cognitive decline in old age. Exp Gerontol 42, 693-701.

Wong, H.L., Pfeiffer, R.M., Fears, T.R., Vermeulen, R., Ji, S., Rabkin, C.S., 2008. Reproducibility and correlations of multiplex cytokine levels in asymptomatic persons. Cancer Epidemiol Biomarkers Prev 17, 3450-3456.

Young, J.J., Bruno, D., Pomara, N., 2014. A review of the relationship between proinflammatory cytokines and major depressive disorder. J Affect Disord 169, 15-20.

Zhou, X., Fragala, M.S., McElhaney, J.E., Kuchel, G.A., 2010. Conceptual and methodological issues relevant to cytokine and inflammatory marker measurements in clinical research. Curr Opin Clin Nutr Metab Care 13, 541-547. 
R. Belzeaux et al.

\section{Figure Legend}

Figure 1. IL-6 correlations between singleplex and multiplex methods and the intra-individual variability across time. Scatterplot of the concentrations of the serum IL-6 analyzed using ELISA and either Luminex ${ }^{\circ}(A ; R h o=-0.061 ; p=0.844)$ or MSD $(B ; R h o=0.781 ; p=4.86 E-$ 5). Scatterplot of the concentrations of the plasma IL-6 analyzed using ELISA and either Luminex ${ }^{\circledR}(C ; R h o=0.330 ; p=0.181)$ or $\operatorname{MSD}(D ; R h o=0.767 ; p=1.16 E-3)$. The intraindividual stability of the serum (E) and plasma (F) IL-6 concentrations measured on the MSD platform at inclusion (V1), and 2 (V2), 8 (V3) and 30 weeks later (V4). 
Reviewer \#3: The reviewer appreciates the responses, however, no fundamental changes have been made to the manuscript in response to my previous critique (except to slightly change the title). My previous points remain unchanged, and cannot be addressed solely in the limitations section of the manuscript.

We regret that our responses could not satisfy totally the reviewer. Nevertheless, we did our best to insist on the limitations of our study by making modifications to our discussion. In addition, very recent results that we mentioned as data not shown, point to a robust inter-assay stability for specific cytokines on the MSD platform. We also got the opportunity to test 2 chemokines on the MSD platform that had been tested on the Luminex platform only in our manuscript. For these chemokines, we obtained very robust correlations between both platforms measurements. All these modifications have been indicated on page 19 of the revised manuscript as follows:

"Our findings have several important technical and theoretical limitations. First, we could not compare exactly the same sets of cytokines on both platforms on the basis of the commercial availability, and it has previously been observed that assay performance decreases with increasing numbers of markers on a panel (Chaturvedi et al., 2011). It will be important in the future to follow up with studies to compare larger sets of cytokines as similar to each other as possible, because recent investigations in the field of biomarker discovery have revealed new candidates for promising proteins. Secondly, we did not address the inter-assay variability on each platform, which is an important factor to consider especially when investigating large samples that cannot be processed with a single batch of reagents or for longitudinal studies when assaying the samples at different days over long periods of time. Third, our results should be considered to be 
preliminary on the basis of the relatively small sample size that makes any group comparison between healthy control and patients inappropriate. Of note, for the purpose of another study, all serum samples assayed in the present study, and obtained from healthy control and MDE patients at their first visit, have been tested again on the MSD platform with a different batch of reagents and a two-year interval (data not shown). The same panels of cytokines were assayed as well as an additional chemokine panel. A comparative analysis of old and new measurements of cytokine concentrations revealed robust correlations for IFN- $y$, IL-6, IL-8, IL-10, IL-12p40, IL-15, IL-16, TNF, TNF- $\beta$, and VEGF (data not shown), suggesting a strong inter-assay stability for these analytes on the MSD platform. Moreover, the new measurements for IP-10 and MIP-1 $\beta$ obtained on the MSD platform strongly correlated with previous measurements obtained with the Luminex platform (data not shown), suggesting that these assays exhibit low intra- and inter-assay variability on both platforms. ” 
R. Belzeaux et al.

\section{Highlights}

We compared two multiplex-cytokine detection technologies

Both platforms performances were unequal depending on tested cytokines

Healthy state is not synonymous of stability in blood circulating cytokines

Caution is required to profile body fluid with multiplex technologies 
R. Belzeaux et al.

\section{Contributors}

R. Belzeaux, J. Boucraut, P. Gressens, N. Glaichenhaus and E.C. Ibrahim designed the study. M. N. Lefebvre recruited the healthy control subjects. R. Belzeaux, X. Zendjidjian, M. Abbar and P. Courtet recruited the patients. R. Belzeaux, J. Naudin, J. Boucraut, P. Gressens, N. Glaichenhaus, and E.C. Ibrahim obtained funding for the study. J. Boucraut and E.C. Ibrahim prepared serum samples. A. Lazzari and T. Le Carpentier performed multiplex assay experiments. R. Belzeaux, J.L Consoloni and E.C. Ibrahim performed statistical analysis. R. Belzeaux, N. Glaichenhaus and E.C. Ibrahim wrote the manuscript. All authors approved the final version of the manuscript. 


\section{Conflict of interest}

The authors have no conflicts of interest to disclose. 
R. Belzeaux et al.

\section{Role of the funding source}

Funding for this study was provided by grant from the French Multi-organization Thematic Institute in Immunology, Hematology and Pneumology (AVIESAN) as well by a national hospital clinical research program (PHRC, No. 2010-19) and by the French Government (National Research Agency, ANR) through the VASPAC project (ANR-13-SAMA-0002) and "Investments for the Future" LABEX SIGNALIFE (\# ANR-11-LABX-0028-01). These funding sources had no further role in study design; in the collection, analysis and interpretation of data; in the writing of the report; and in the decision to submit the paper for publication. 
R. Belzeaux et al.

\section{Acknowledgments}

The authors acknowledge support by Fondation Fondamental, INSERM, AP-HM, AixMarseille University, Université Paris Diderot and Université de Nice-Sophia Antipolis. We thank Prof. Marion Leboyer for inspiring the present work by connecting and providing constant support to investigators interested by the emerging field of Immunopsychiatry. We also thank Jeanne Hsu for editing the manuscript. 
Table1 Dynamic range and intra-assay CVs of the standard calibrators on the Luminex and MSD platforms.

\begin{tabular}{|c|c|c|c|c|c|c|c|c|}
\hline \multirow[t]{2}{*}{ Analyte } & \multicolumn{4}{|c|}{ Luminex } & \multicolumn{4}{|c|}{ MSD } \\
\hline & $\begin{array}{l}\text { LLOQ-ULOQ } \\
(\mathrm{pg} / \mathrm{ml})\end{array}$ & $\begin{array}{l}\text { detection } \\
\text { range }\end{array}$ & $\begin{array}{l}\mathrm{CV} \\
(\%)\end{array}$ & $\begin{array}{l}\text { linearity } \\
(\%)\end{array}$ & $\begin{array}{l}\text { LLOQ-ULOQ } \\
(\mathrm{pg} / \mathrm{ml})\end{array}$ & $\begin{array}{l}\text { detection } \\
\text { range }\end{array}$ & $\begin{array}{l}\text { CV } \\
(\%)\end{array}$ & $\begin{array}{l}\text { linearity } \\
(\%)\end{array}$ \\
\hline Eotaxin & $1.72-7047$ & 4097 & 4.5 & 91.1 & & & & \\
\hline bFGF & $14.3-14649$ & 1024 & 3.4 & 104 & & & & \\
\hline G-CSF & $1.73-51920$ & 30012 & 4.1 & 91.1 & & & & \\
\hline IL-1RA & $1.85-121425$ & 65635 & 5.6 & 81.8 & & & & \\
\hline IL-9 & $1.24-22978$ & 18531 & 4.0 & 79.3 & & & & \\
\hline IP-10 & $1.3-21311$ & 16393 & 4.1 & 95.3 & & & & \\
\hline MCP-1 & $13.5-27412$ & 2030 & 3.6 & 73.7 & & & & \\
\hline MIP-1 $\alpha$ & $0.58-2387$ & 4116 & 2.7 & 94.0 & & & & \\
\hline MIP-1 $\beta$ & $1.11-6448$ & 5809 & 2.7 & 88.1 & & & & \\
\hline PDGF-BB & $1.07-20331$ & 19001 & 5.2 & 87.1 & & & & \\
\hline RANTES & $3.62-14838$ & 4099 & 3.3 & 103 & & & & \\
\hline IL-1 $\alpha$ & & & & & $0.09-405$ & 4500 & 5.7 & 97.1 \\
\hline IL-12p40 & & & & & $0.75-3220$ & 4293 & 3.9 & 98.2 \\
\hline IL-16 & & & & & $0.62-2810$ & 4532 & 4.4 & 122 \\
\hline TNF- $\beta$ & & & & & $0.14-632$ & 4514 & 3.3 & 103 \\
\hline GM-CSF & $0.66-13149$ & 19923 & 3.5 & 74.9 & $0.23-996$ & 4330 & 4.7 & 96.8 \\
\hline IFN- & $3.62-34365$ & 9093 & 4.5 & 100 & $0.33-1460$ & 4424 & 4.0 & 92.5 \\
\hline$\overline{\mathrm{IL}-1 \boldsymbol{\beta}}$ & $0.08-7731$ & 96638 & 4.8 & 86.8 & $0.12-495$ & 4125 & 3.6 & 94.6 \\
\hline IL-2 & $4.75-19450$ & 4095 & 2.2 & 71.7 & $0.32-1460$ & 4563 & 3.3 & 93.2 \\
\hline IL-4 & $0.2-4149$ & 20745 & 4.7 & 94.5 & $0.05-262$ & 5240 & 6.4 & 97.2 \\
\hline$\underline{\mathrm{IL}-5}$ & $0.8-13069$ & 16336 & 4.8 & 108 & $0.20-912$ & 4560 & 4.6 & 95.3 \\
\hline IL-6 & $1.41-26740$ & 18965 & 2.7 & 80.3 & $0.16-767$ & 4794 & 3.1 & 108 \\
\hline$\overline{I L-7}$ & $0.26-16792$ & 64590 & 5.7 & 95.7 & $0.15-838$ & 5587 & 5.4 & 102 \\
\hline$\underline{\mathrm{IL}-8}$ & $0.35-39280$ & 112229 & 5.0 & 79.2 & $0.12-500$ & 4167 & 3.4 & 97.1 \\
\hline IL-10 & $3.39-35130$ & 10363 & 3.1 & 75.5 & $0.08-334$ & 4175 & 4.5 & 92.4 \\
\hline IL-12p70 & $2.33-38575$ & 16556 & 4.6 & 77.5 & $0.10-421$ & 4210 & 4.0 & 92.8 \\
\hline IL-13 & $0.43-6976$ & 16220 & 3.8 & 86.5 & $0.61-626$ & 1026 & 4.2 & 133 \\
\hline$\overline{\mathrm{IL}-15}$ & $6.49-6647$ & 1024 & 4.9 & 63.3 & $0.17-779$ & 4582 & 4.0 & 102 \\
\hline$\overline{\mathrm{LL}-17}$ & $6.16-32716$ & 5311 & 3.1 & 82.9 & $1.38-6010$ & 4355 & 4.9 & 103 \\
\hline TNF & $0.93-60786$ & 65361 & 3.7 & 88.9 & $0.07-317$ & 4529 & 5.1 & 95.6 \\
\hline VEGF & $2.37-38806$ & 16374 & 3.7 & 75.9 & $0.24-1020$ & 4250 & 5.1 & 121 \\
\hline
\end{tabular}

$\mathrm{CV}<5 \%$ are indicated in bold.

Analytes with $\mathrm{CV}<5 \%$ in at least one platform are indicated in bold. Analytes with $\mathrm{CV}<5 \%$ in both platforms are underlined. 
Table 2 Proportion of serum samples within the detection range and intra-assay variability.

\begin{tabular}{|c|c|c|c|c|c|c|}
\hline & \multicolumn{4}{|c|}{$\begin{array}{c}\% \text { of samples within the detection } \\
\text { range }\end{array}$} & \multicolumn{2}{|c|}{$\begin{array}{c}\text { Intra-assay CV } \\
\text { (\%) }\end{array}$} \\
\hline & \multicolumn{2}{|c|}{ Luminex } & \multicolumn{2}{|c|}{ MSD } & Luminex & MSD \\
\hline & Patient & Control & Patient & Control & & \\
\hline Eotaxin & 100 & 100 & & & 5.86 & \\
\hline bFGF & 12.5 & 11.1 & & & ND & \\
\hline G-CSF & 77.5 & 91.7 & & & 11.3 & \\
\hline$\overline{\text { IL-1RA }}$ & 90.0 & 55.6 & & & 15.3 & \\
\hline$\underline{\text { IL-9 }}$ & 100 & 100 & & & 13.6 & \\
\hline IP-10 & 100 & 100 & & & 3.58 & \\
\hline$\overline{\mathrm{MCP}-1}$ & 2.50 & 0 & & & ND & \\
\hline MIP-1 $\alpha$ & 40.0 & 47.2 & & & 16.1 & \\
\hline MIP-1 $\beta$ & 100 & 100 & & & 4.04 & \\
\hline PDGF-BB & 100 & 100 & & & 4.99 & \\
\hline RANTES & 75.0 & 58.3 & & & 9.05 & \\
\hline IL-1 $\alpha$ & & & 50.0 & 44.7 & & 31.9 \\
\hline IL-12p40 & & & 100 & 100 & & 4.83 \\
\hline IL-16 & & & 100 & 100 & & 4.27 \\
\hline$\overline{T N F-\beta}$ & & & 65.0 & 71.1 & & 15.5 \\
\hline GM-CSF & 0 & 0 & 17.5 & 5.26 & ND & ND \\
\hline IFN-Y & 0 & 0 & 100 & 100 & ND & 4.78 \\
\hline$\overline{\mathrm{IL}-1 \beta}$ & 57.5 & 58.3 & 40.0 & 44.7 & 28.5 & 57.7 \\
\hline IL-2 & 27.5 & 36.1 & 7.5 & 15.8 & ND & ND \\
\hline IL-4 & 100 & 100 & 17.5 & 15.8 & 17.5 & ND \\
\hline IL-5 & 40.0 & 47.2 & 27.5 & 13.2 & 15.4 & ND \\
\hline$\underline{\mathrm{IL}-6}$ & 72.5 & 36.1 & 95.0 & 94.7 & 19.7 & 11.3 \\
\hline$\underline{\text { IL-7 }}$ & 100 & 88.9 & 100 & 100 & 20.1 & 5.13 \\
\hline$\underline{\mathrm{IL}-8}$ & 100 & 100 & 100 & 100 & 10.5 & 4.49 \\
\hline$\underline{\mathrm{IL}-10}$ & 70.0 & 69.4 & 100 & 100 & 12.8 & 10.0 \\
\hline IL-12p70 & 100 & 100 & 35.0 & 26.3 & 11.2 & ND \\
\hline IL-13 & 100 & 100 & 34.9 & 10.5 & 14.4 & ND \\
\hline IL-15 & 0 & 0 & 100 & 100 & ND & 3.37 \\
\hline$\overline{I L-17}$ & 77.5 & 83.3 & 60.0 & 42.1 & 21.8 & 19.2 \\
\hline$\underline{\text { TNF }}$ & 97.5 & 100 & 97.5 & 100 & 14.1 & 5.86 \\
\hline VEGF & 97.5 & 100 & 100 & 100 & 11.0 & 3.02 \\
\hline Total & $18 / 27$ & $15 / 27$ & $12 / 20$ & $11 / 20$ & $13 / 27$ & $10 / 20$ \\
\hline
\end{tabular}

Analytes that were above the LLOQ in at least $60 \%$ of serum samples are indicated in bold. Among these cytokines, those exhibiting $\mathrm{CVs} \leq 15 \%$ on at least one platform are underlined. CVs were not determined (ND) for cytokines that were above the LLOQ in less than $40 \%$ of both control and patient samples. 
Table 3 Proportion of plasma samples within the detection range and intra-assay variability.

\begin{tabular}{|c|c|c|c|c|c|c|}
\hline & \multicolumn{4}{|c|}{$\begin{array}{c}\% \text { of samples within the detection } \\
\text { range }\end{array}$} & \multicolumn{2}{|c|}{$\begin{array}{c}\text { Intra-assay CV } \\
\text { (\%) }\end{array}$} \\
\hline & \multicolumn{2}{|c|}{ Luminex } & \multicolumn{2}{|c|}{ MSD } & \multirow{2}{*}{ Luminex } & \multirow[t]{2}{*}{ MSD } \\
\hline & Patient & Control & Patient & Control & & \\
\hline Eotaxin & 100 & 100 & & & 5.58 & \\
\hline bFGF & 100 & 95.8 & & & 7.43 & \\
\hline$\overline{\text { G-CSF }}$ & 100 & 100 & & & 19.8 & \\
\hline IL-1RA & 100 & 100 & & & 5.68 & \\
\hline$\underline{\text { IL-9 }}$ & 100 & 100 & & & 11.3 & \\
\hline$\overline{\text { IP-10 }}$ & 100 & 100 & & & 6.12 & \\
\hline$\overline{\mathrm{MCP}-1}$ & 13.8 & 13.9 & & & ND & \\
\hline MIP-1 $\alpha$ & 100 & 100 & & & 5.36 & \\
\hline MIP-1 $\beta$ & 100 & 100 & & & 3.92 & \\
\hline PDGF-BB & 100 & 100 & & & 6.10 & \\
\hline RANTES & 100 & 97.2 & & & 4.68 & \\
\hline IL-1 $\alpha$ & & & 77.5 & 62.5 & & 26.9 \\
\hline IL-12p40 & & & 100 & 100 & & 4.36 \\
\hline IL-16 & & & 100 & 100 & & 4.29 \\
\hline TNF- $\boldsymbol{\beta}$ & & & 76.3 & 80.0 & & 16.6 \\
\hline GM-CSF & 90 & 97.2 & 31.3 & 32.5 & 17.6 & ND \\
\hline IFN- & 100 & 100 & 100 & 100 & 7.53 & 10.9 \\
\hline$\overline{\mathrm{IL}-1 \beta}$ & 100 & 100 & 10.0 & 7.50 & 6.83 & ND \\
\hline IL-2 & 0 & 0 & 2.50 & 18.8 & ND & ND \\
\hline IL-4 & 100 & 100 & 2.50 & 0.00 & 7.86 & ND \\
\hline IL-5 & 100 & 100 & 86.3 & 82.5 & 8.95 & 20.1 \\
\hline$\underline{\mathrm{IL}-6}$ & 82.5 & 90.3 & 78.8 & 83.8 & 12.0 & 10.9 \\
\hline$\overline{\text { IL-7 }}$ & 100 & 100 & 100 & 100 & 9.16 & 7.68 \\
\hline$\overline{\mathrm{IL}-8}$ & 100 & 100 & 100 & 100 & 9.11 & 5.57 \\
\hline IL-10 & 92.5 & 94.4 & 86.3 & 85.0 & 9.98 & 16.6 \\
\hline IL-12p70 & 97.5 & 98.6 & 22.5 & 7.50 & 11.5 & ND \\
\hline$\underline{\text { IL-13 }}$ & 100 & 100 & 32.5 & 42.5 & 5.26 & ND \\
\hline$\overline{\text { IL-15 }}$ & 41.2 & 61.1 & 100 & 100 & 17.2 & 3.99 \\
\hline$\underline{\text { IL-17 }}$ & 100 & 98.6 & 57.5 & 45.0 & 8.93 & 17.3 \\
\hline$\overline{\mathrm{TNF}}$ & 100 & 100 & 100 & 98.8 & 7.18 & 10.1 \\
\hline VEGF & 95.0 & 100 & 100 & 100 & 9.76 & 4.76 \\
\hline Total & $24 / 27$ & $25 / 27$ & $13 / 20$ & $13 / 20$ & $22 / 27$ & $9 / 20$ \\
\hline
\end{tabular}

Analytes that were above the LLOQ in at least $60 \%$ of either control or patient plasma samples are indicated in bold. Among these cytokines, those exhibiting CVs $\leq 15 \%$ on at least one platform are underlined. CVs were not determined (ND) for cytokines that were above the LLOQ in less than $40 \%$ of both control and patient samples. 
Table 4 Correlation of measurements between sera and plasma samples.

\begin{tabular}{|c|c|c|c|c|}
\hline & \multicolumn{2}{|c|}{ Luminex } & \multicolumn{2}{|c|}{ MSD } \\
\hline & Rho & $p$-value & Rho & $p$-value \\
\hline Eotaxin & 0.902 & $5.40 \mathrm{E}-8$ & & \\
\hline$\overline{\text { IL-1RA }}$ & -0.072 & 7.83E-1 & & \\
\hline IL-9 & 0.415 & $6.90 \mathrm{E}-2$ & & \\
\hline IP-10 & 0.767 & 7.98E-5 & & \\
\hline MIP-1 $\beta$ & 0.710 & 8.00E-5 & & \\
\hline PDGF-BB & 0.206 & $3.84 \mathrm{E}-1$ & & \\
\hline RANTES & 0.464 & $8.13 E-2$ & & \\
\hline IL-12p40 & & & 0.860 & 1.16E-6 \\
\hline IL-16 & & & 0.415 & $6.88 \mathrm{E}-2$ \\
\hline IFN- $\nu$ & & & 0.967 & 4.10E-12 \\
\hline IL-4 & 0.162 & $4.94 \mathrm{E}-1$ & & \\
\hline IL-6 & 0.063 & $8.46 \mathrm{E}-1$ & 0.838 & 5.01E-5 \\
\hline IL-7 & -0.133 & $5.75 \mathrm{E}-1$ & -0.200 & $5.80 \mathrm{E}-1$ \\
\hline IL-8 & 0.116 & $6.28 \mathrm{E}-1$ & -0.205 & $3.87 \mathrm{E}-1$ \\
\hline IL-10 & 0.556 & 1.11E-2 & 0.564 & $1.84 \mathrm{E}-2$ \\
\hline IL-12p70 & 0.290 & $2.14 \mathrm{E}-1$ & & \\
\hline IL-13 & 0.797 & 2.60E-5 & & \\
\hline IL-15 & & & 0.750 & $1.38 \mathrm{E}-4$ \\
\hline TNF & 0.093 & $6.98 \mathrm{E}-1$ & 0.684 & 8.77E-4 \\
\hline VEGF & 0.174 & 4.77E-1 & 0.535 & $1.50 \mathrm{E}-2$ \\
\hline Total & & $5 / 16$ & & $7 / 10$ \\
\hline
\end{tabular}

Cytokines that exhibited significant correlation in either platform according to Spearman non-

parametric test were indicated in bold. 
Table 5 Correlation of measurements between the Luminex and MSD platforms.

\begin{tabular}{llllll}
\hline & \multicolumn{2}{c}{ Serum } & & \multicolumn{2}{c}{ Plasma } \\
\cline { 6 - 6 } \cline { 5 - 6 } & Rho & p-value & & Rho & p-value \\
\hline IFN- $\gamma$ & & & & -0.229 & $3.32 \mathrm{E}-1$ \\
IL-6 & & & & $\mathbf{0 . 6 3 2}$ & $\mathbf{1 . 1 5 E}-\mathbf{2}$ \\
IL-7 & & & & 0.236 & $5.11 \mathrm{E}-1$ \\
IL-8 & $\mathbf{0 . 8 9 8}$ & $\mathbf{7 . 7 4 E}-\mathbf{8}$ & & -0.202 & $3.92 \mathrm{E}-1$ \\
IL-10 & $\mathbf{0 . 0 9 8}$ & $\mathbf{6 . 8 2 E}-1$ & & 0.145 & $5.80 \mathrm{E}-1$ \\
TNF & -0.273 & $2.44 \mathrm{E}-1$ & & 0.289 & $2.17 \mathrm{E}-1$ \\
VEGF & $\mathbf{0 . 8 7 1}$ & $\mathbf{5 . 9 5 E - 7}$ & & $\mathbf{0 . 7 3 0}$ & $\mathbf{3 . 9 0 E}-\mathbf{4}$ \\
\hline
\end{tabular}

Cytokines that exhibited significant correlation in either platform according to Spearman non-

parametric test were indicated in bold. 
Table 6 Variability of analyte concentration in healthy subjects during a 30-week period.

\begin{tabular}{lllll}
\hline & \multicolumn{2}{c}{ Serum } & \multicolumn{2}{c}{ Plasma } \\
Eotaxin & Luminex & MSD & Luminex & MSD \\
bFGF & ND & & $\mathbf{0 . 9 7 4}$ & \\
G-CSF & $\mathbf{0 . 7 3 1}$ & & $\mathbf{0 . 9 5 8}$ & \\
IL-1RA & ND & & $\mathbf{0 . 8 0 0}$ & \\
IL-9 & $\mathbf{0 . 9 0 2}$ & & 0.033 & \\
IP-10 & 0.265 & & $\mathbf{0 . 8 9 8}$ & \\
MIP-1 $\boldsymbol{\alpha}$ & ND & & 0.140 & \\
MIP-1 $\beta$ & $\mathbf{0 . 9 1 3}$ & & $\mathbf{0 . 9 6 9}$ & \\
PDGF-BB & $\mathbf{0 . 8 7 3}$ & & 0.736 & \\
RANTES & ND & & 0.468 & \\
IL-12p40 & & $\mathbf{0 . 8 7 4}$ & $\mathbf{0 . 8 9 9}$ & \\
IL-16 & & $\mathbf{0 . 7 6 3}$ & & $\mathbf{0 . 8 2 6}$ \\
TNF- $\beta$ & & $-0,02^{\mathrm{a}}$ & & 0.032 \\
GM-CSF & ND & ND & $\mathbf{0 . 8 6 0}$ & ND \\
IFN- & ND & -0.02 & $\mathbf{0 . 9 7 3}$ & 0.068 \\
IL-1 $\beta$ & ND & ND & $\mathbf{0 . 9 6 0}$ & ND \\
IL-4 & $\mathbf{0 . 8 0 4}$ & ND & $\mathbf{0 . 9 0 1}$ & ND \\
IL-5 & ND & ND & $\mathbf{0 . 9 6 5}$ & ND \\
IL-6 & ND & 0.304 & $\mathbf{0 . 9 5 4}$ & 0.044 \\
IL-7 & ND & $\mathbf{0 . 9 2 1}$ & $\mathbf{0 . 9 4 6}$ & $\mathbf{0 . 6 0 8}$ \\
IL-8 & 0.049 & 0.068 & $\mathbf{0 . 8 9 4}$ & $-0.01^{\text {a }}$ \\
IL-10 & 0.140 & $-0.06^{\text {a }}$ & $\mathbf{0 . 9 6 2}$ & 0.105 \\
IL-12p70 & $\mathbf{0 . 8 3 3}$ & ND & $\mathbf{0 . 8 9 7}$ & ND \\
IL-13 & 0.538 & ND & $\mathbf{0 . 8 8 6}$ & ND \\
IL-15 & ND & $\mathbf{0 . 6 7 7}$ & $\mathbf{0 . 9 5 0}$ & $\mathbf{0 . 7 8 8}$ \\
IL-17 & ND & 0.469 & $\mathbf{0 . 9 5 4}$ & \\
TNF & 0.425 & $\mathbf{0 . 8 6 7}$ & $\mathbf{0 . 9 5 6}$ & $\mathbf{0 . 7 3 7}$ \\
VEGF & $\mathbf{0 . 8 8 8}$ & $\mathbf{0 . 9 7 4}$ & 0.510 & 0.447 \\
\hline SOme neg & & & \\
\hline
\end{tabular}

${ }^{\mathrm{a}}$ Some negative values for the ICC should be interpreted as providing poor agreement (Giraudeau, 1996). Analytes in bold indicate the intra-indivdual stability considering an ICC $\geq 0.55$ for both the sera and plasma samples with either one or both platforms. Statistical tests were not determined (ND) for analytes that were either above the LLOQ in less than $40 \%$ of control samples or that provided CV of duplicates $>20 \%$. 

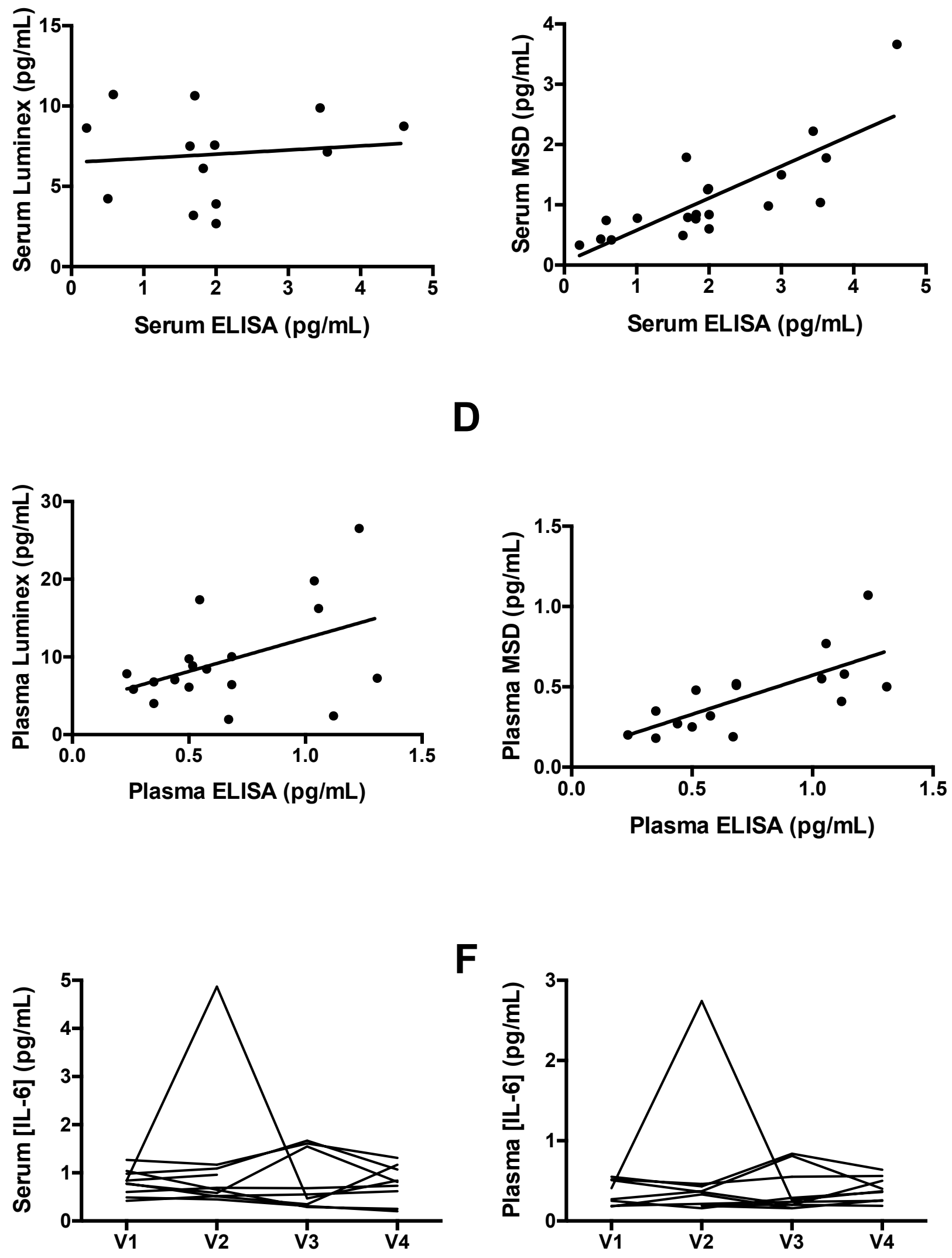
Supplementary Table 1

Click here to download Optional e-only supplementary files: SupTab1_130916.docx

Click here to download Optionale-only supplementary files: SupTab1_130916.docx 
Supplementary Table 2

Click here to download Optional e-only supplementary files: SupTab2_130916.docx

Click here to download Optionale-only supplementary files: SupTab2_130916.docx

(n)


Supplementary Table 3
Click here to download Optional e-only supplementary files: SupTab3_130916.docx

Click here to download Optional e-only supplementary files: SupTab3_130916.docx

(a)


Supplementary Table 4
Click here to download Optional e-only supplementary files: SupTab4_130916.docx

Click here to download Optional e-only supplementary files: SupTab4_130916.docx

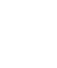


Supplementary Table 5
Click here to download Optional e-only supplementary files: SupTab5_130916.docx 
Supplementary Table 6
Click here to download Optional e-only supplementary files: SupTable6_130916.docx

Click here to download Optional e-only supplementary files: SupTable6_130916.docx 
Supplementary Figure 1
Click here to download Optional e-only supplementary files: SupFig1_26082016.pptx

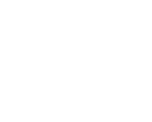

$\sqrt{2}$

$\sqrt{2}$

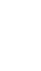

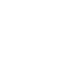

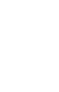
(n)

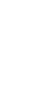

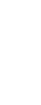
(1)

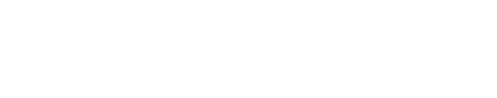

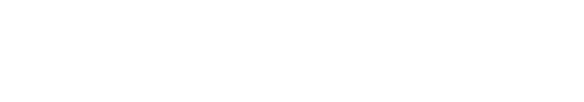
(n) (1)

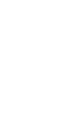

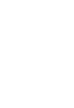

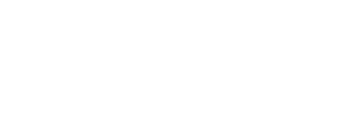

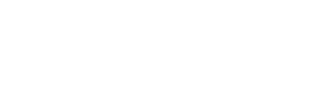


Supplementary Figure 2
Click here to download Optional e-only supplementary files: SupFigure 2_legende_130916.docx

Figure 2_legende_130916.docx

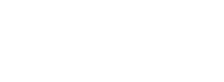

ptional e-only supple

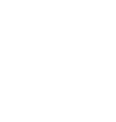

$\sqrt{2}$

(1)

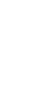
(1)

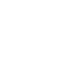
(1) (1) (1) (1)

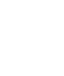
(1)

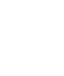
. . . . . . . . . . 


\section{Click here to download Optional e-only supplementary files: SupFigure 3_legende_290816.docx}

